\title{
Weyl-type topological phase transitions in fractional quantum Hall like systems
}

\author{
Stefanos Kourtis, ${ }^{1}$ Titus Neupert, ${ }^{2}$ Christopher Mudry, ${ }^{3}$ Manfred Sigrist, ${ }^{4}$ and Wei Chen ${ }^{4}$ \\ ${ }^{1}$ Department of Physics, Boston University, Boston, Massachusetts 02215, USA \\ ${ }^{2}$ Department of Physics, University of Zurich, Winterthurerstrasse 190, CH-8057 Zurich, Switzerland \\ ${ }^{3}$ Condensed Matter Theory Group, Paul Scherrer Institute, CH-5232 Villigen PSI, Switzerland \\ ${ }^{4}$ Institute for Theoretical Physics, ETH Zurich, CH-8093 Zurich, Switzerland
}

(Received 18 August 2017; revised manuscript received 30 October 2017; published 10 November 2017)

\begin{abstract}
We develop a method to characterize topological phase transitions for strongly correlated Hamiltonians defined on two-dimensional lattices based on the many-body Berry curvature. Our goal is to identify a class of quantum critical points between topologically nontrivial phases with fractionally quantized Hall (FQH) conductivity and topologically trivial gapped phases through the discontinuities of the many-body Berry curvature in the so-called flux Brillouin zone (fBZ), the latter being defined by imposing all possible twisted boundary conditions. For this purpose, we study the finite-size signatures of several quantum phase transitions between fractional Chern insulators and charge-ordered phases for two-dimensional lattices by evaluating the many-body Berry curvature numerically using exact diagonalization. We observe degeneracy points (nodes) of many-body energy levels at high-symmetry points in the fBZ, accompanied by diverging Berry curvature. We find a correspondence between the number and order of these nodal points, and the change of the topological invariants of the many-body ground states across the transition, in close analogy with Weyl nodes in noninteracting band structures. This motivates us to apply a scaling procedure, originally developed for noninteracting systems, for the Berry curvature at the nodal points. This procedure offers a useful tool for the classification of topological phase transitions in interacting systems harboring FQH like topological order.
\end{abstract}

DOI: 10.1103/PhysRevB.96.205117

\section{INTRODUCTION}

Degeneracies in quantum systems can be accidental or enforced by symmetries. In the former case, slight perturbations applied to the system can in principle remove the degeneracy. Choosing words more carefully, one should distinguish between moving and removing a degeneracy. To elucidate this point, we consider a pair of linearly independent quantum states $\left|\Psi_{1}(\boldsymbol{M})\right\rangle$ and $\left|\Psi_{2}(\boldsymbol{M})\right\rangle$ that are parametrized by a multidimensional vector of real parameters $\boldsymbol{M}$. The matrix elements of a given Hamiltonian with the two states $\left|\Psi_{1}(\boldsymbol{M})\right\rangle$ and $\left|\Psi_{2}(\boldsymbol{M})\right\rangle$ form a $2 \times 2$ Hermitian matrix, and are thus characterized by four real functions of $\boldsymbol{M}$, e.g., as prefactors when expanding in the three Pauli matrices together with the unit $2 \times 2$ matrix. The states are degenerate if all three functions multiplying the three Pauli matrices are tuned to zero at some $\boldsymbol{M}_{\mathrm{c}}$. Such simultaneous zeros will occur generically, if the parameter space in which $\boldsymbol{M}$ lives is three dimensional, in which case perturbations applied to such accidental degeneracies do not remove them but move them in parameter space. If the dimension of parameter space $d$ is smaller than three, three independent real functions cannot be simultaneously tuned to zero, while if it is larger than three, accidental degeneracies will occur on $(d-3)$-dimensional hypersurfaces. These considerations go back to von Neumann and Wigner [1], while Herring [2] and Blount [3] applied this reasoning to band structures of three-dimensional crystals, in which the quasimomentum $\boldsymbol{k}$ takes the role of the parameter vector $\boldsymbol{M}$. Berry and Wilkinson also found in Ref. [4] such degeneracies for quantum singleparticle Hamiltonians without any symmetry. They called such degeneracies diabolical points.

Crucially, these accidental degeneracies found in threedimensional parameter space are topological objects with a quantized chiral charge, so-called Weyl points. The terminology "Weyl point" can be motivated as follows. Consider the two-dimensional Hilbert space spanned by the state $\left|\Psi_{1}(\boldsymbol{M})\right\rangle$ and $\left|\Psi_{2}(\boldsymbol{M})\right\rangle$ whereby both states undergo a level crossing at a diabolical point in parameter space. We may associate to any point in parameter space that is sufficiently close to the diabolical point an Abelian gauge field, the Abelian Berry connection $\left\langle\Psi_{1}(\boldsymbol{M})\left|\mathrm{i} \partial_{\boldsymbol{M}}\right| \Psi_{1}(\boldsymbol{M})\right\rangle$ of state $\left|\Psi_{1}(\boldsymbol{M})\right\rangle$, say. The rotation of this Abelian Berry connection delivers an Abelian magnetic field. The Abelian magnetic flux through any surface that encloses the diabolical point is quantized and thus robust to small and smooth changes in the Hamiltonian [5]. Any such diabolical point is therefore a topological object with a quantized charge, i.e., a monopole for an Abelian Berry magnetic flux. Because the four matrix elements of the Hamiltonian between these two states realize a Weyl Hamiltonian close to a diabolical point, we shall rename this point in parameter space a Weyl point. Generic perturbations will simply move a Weyl point in parameter space, but cannot eliminate it.

In this paper, we study numerically away from the thermodynamic limit Weyl points associated with degeneracies between two many-body quantum states close to a topological phase transition occurring in parameter space in the thermodynamic limit. Away from multicritical points, phase transitions are driven by a single external parameter (i.e., the codimension of the phase boundary in parameter space is one). It is then most natural to look for phase transitions characterized by accidental Weyl-type degeneracies of two quantum states in two-dimensional systems because the phase angles of twisted boundary conditions comprise two parameters that add up with a control parameter in the many-body interacting Hamiltonian to a three-dimensional parameter space. In this case, the topological charge associated with the degeneracy is nothing but the change in the Hall conductivity averaged over twisted boundary conditions following the formula of Niu and Thouless [6,7]. This change has a finite-size signature as it is unambiguously determined by a single many-body level 
crossing for the many-body interacting lattice Hamiltonian, whereby the lattice is of finite size. The main result of this paper is that, in close analogy to noninteracting band structures over a three-dimensional Brillouin zone, the change in the Hall conductivity of any two-dimensional many-body interacting lattice Hamiltonian subject to twisted boundary conditions across a quantum phase transition controlled by one real parameter equals the topological charge associated with a many-body Weyl point between two many-body eigenstates.

Weyl-type quantum phase transitions are thus necessarily topological quantum phase transitions and one may expect them to be realized in systems that support, in one phase, a (fractional) quantum Hall effect. This is also in agreement with the fact that quantum Hall systems do not rely on any symmetries (aside from charge conservation), just as a Weyl point of unit chiral charge is not stabilized by any symmetries.

Our approach to Weyl-type quantum phase transitions is focused on numerical investigation of strongly interacting finitesized systems. To easily apply twisted boundary conditions, we study lattice systems, and in particular the transition between lattice realizations of the fractional quantum Hall effect, that is, fractional Chern insulators (FCIs) [8,9], and charge-ordered phases. We demonstrate that these quantum phase transitions are indeed of Weyl type. We show examples for both bosonic and fermionic systems and present a case in which lattice symmetries give rise to a Weyl point of higher charge (two) at the phase transition, which splits into two Weyl points of unit charge if these symmetries are broken. Furthermore, we point out the similarities and differences between the physics of Weyl points occurring between many-body states and those in noninteracting band structures, and apply a scaling procedure that captures the numerically observed phenomenology of the Weyl-type quantum phase transitions.

This paper is organized as follows. We review the NiuThouless formula in Sec. II. We define lattice models for interacting fermions or bosons that undergo a quantum phase transition between a fractional Chern insulating phase and a trivial one (i.e., a phase with vanishing Hall conductivity) and show numerically the existence of Weyl (diabolical) points in parameter space in Sec. III. We verify that a scaling analysis for the many-body Berry curvature applies in Sec. IV. We conclude with Sec. V.

\section{BERRY CURVATURE AND CHERN NUMBER OF MANY-BODY STATES}

Consider a two-dimensional system of $N$ interacting identical quantum particles on a lattice made of $L_{1} \times L_{2}$ unit cells, defined by the primitive translation vectors $\boldsymbol{a}_{1}$ and $\boldsymbol{a}_{2}$. The system is described by a many-body Hamiltonian $\widehat{H}(\boldsymbol{M})$ that depends on a number of parameters with units of energy $\boldsymbol{M} \equiv\left(M_{1}, \ldots, M_{m}\right)^{\top} \in \mathbb{R}^{m}$. We also impose twisted periodic boundary conditions on the system. This amounts to introducing a second parametric dependence of the Hamiltonian on the twisting angles given by the vector $\phi^{\top}=\left(\phi_{1}, \phi_{2}\right)$, i.e., $\widehat{H} \equiv \widehat{H}(\boldsymbol{\phi}, \boldsymbol{M})$. Hence, all many-body states $|\Psi(\boldsymbol{\phi}, \boldsymbol{M})\rangle$ obey the twisted boundary conditions

$$
\begin{aligned}
& \widehat{T}_{i, L_{1} a_{1}}|\Psi(\boldsymbol{\phi}, \boldsymbol{M})\rangle=e^{\mathrm{i} \boldsymbol{\phi}_{1}}|\Psi(\boldsymbol{\phi}, \boldsymbol{M})\rangle, \\
& \widehat{T}_{i, L_{2} \boldsymbol{a}_{2}}|\Psi(\phi, \boldsymbol{M})\rangle=e^{\mathrm{i} \phi_{2}}|\Psi(\boldsymbol{\phi}, \boldsymbol{M})\rangle,
\end{aligned}
$$

where $\widehat{T}_{i, \boldsymbol{r}}$ is the operator that translates particle $i=1, \ldots, N$ by $\boldsymbol{r}$.

The Hall conductivity $\sigma_{\mathrm{H}}(\boldsymbol{\phi}, \boldsymbol{M})$ at zero temperature governs the transverse linear response to the infinitesimal variation $\boldsymbol{\phi} \rightarrow \boldsymbol{\phi}+\delta \boldsymbol{\phi}$. It is given by $[6,10]$

$$
\sigma_{\mathrm{H}}(\boldsymbol{\phi}, \boldsymbol{M})=\frac{e^{2}}{h} \frac{1}{N_{g=1}} \sum_{n=1}^{N_{g=1}} F_{n}(\boldsymbol{\phi}, \boldsymbol{M}) .
$$

Here, $e$ is the electric charge, $h$ is the Planck constant, and the integer $N_{g=1}$ is the ground-state (GS) degeneracy in the thermodynamic limit upon imposing the twisted boundary conditions $\boldsymbol{\phi}$. Moreover, the function $F_{n}(\boldsymbol{\phi}, \boldsymbol{M})$ is usually defined through linear response theory by the Kubo formula

$$
F_{n}(\boldsymbol{\phi}, \boldsymbol{M}):=4 \pi \operatorname{Im} \sum_{n^{\prime} \neq n} \frac{\left\langle\Psi_{n}\left|\partial_{\phi_{2}} \widehat{H}\right| \Psi_{n^{\prime}}\right\rangle\left\langle\Psi_{n^{\prime}}\left|\partial_{\phi_{1}} \widehat{H}\right| \Psi_{n}\right\rangle}{\left(E_{n^{\prime}}-E_{n}\right)^{2}}
$$

provided all denominators on the right-hand side are nonvanishing in a sufficiently small open set containing $\phi$ and $\boldsymbol{M}$. Here, the summation over $n^{\prime}$ runs over all the eigenstates $\left|\Psi_{n^{\prime}}(\boldsymbol{\phi}, \boldsymbol{M})\right\rangle$ of $\widehat{H}(\boldsymbol{\phi}, \boldsymbol{M})$ that are orthogonal to any one of the GSs $\left|\Psi_{n}(\boldsymbol{\phi}, \boldsymbol{M})\right\rangle$ with $n=1, \ldots, N_{g=1}$. Their eigenenergies are $E_{n^{\prime}}(\boldsymbol{\phi}, \boldsymbol{M})$ and $E_{n}(\boldsymbol{\phi}, \boldsymbol{M})$, respectively. In the thermodynamic limit and on the torus (genus $g=1$ ) over which the twisted boundary conditions are imposed, the eigenenergies $E_{n}(\boldsymbol{\phi}, \boldsymbol{M})$, with $n=1, \ldots, N_{g=1}$, are all degenerate. For notational simplicity, we have dropped the explicit dependence of the Hamiltonian with its eigenenergies and eigenstates on $\boldsymbol{\phi}$ and $\boldsymbol{M}$ in Eq. (2.2b). The Hall conductivity (2.2a) is averaged over all states in the manifold of $N_{g=1}$-fold degenerate GS when two-dimensional space is the twisted torus and the thermodynamic limit is defined by taking the limit $L_{1}, L_{2} \rightarrow \infty$ while holding the particle density $N /\left(L_{1} L_{2}\right)$ fixed.

The Hall conductivity at zero temperature should only depend on the linearly independent GSs of the many-body interacting Hamiltonian $\widehat{H}$. This fact is not explicit in Eqs. (2.2a) and (2.2b) as all many-body excited states and their eigenvalues contribute to the Hall conductivity. It turns out that the quantity $F_{n}(\boldsymbol{\phi}, \boldsymbol{M})$ is called the many-body Berry curvature of $\left|\Psi_{n}(\boldsymbol{\phi}, \boldsymbol{M})\right\rangle$ as it can be expressed in terms of the partial derivatives of $\left|\Psi_{n}(\boldsymbol{\phi}, \boldsymbol{M})\right\rangle$ according to the formula $[6,10]$

$$
F_{n}(\boldsymbol{\phi}, \boldsymbol{M})=4 \pi \operatorname{Im}\left\langle\frac{\partial \Psi_{n}}{\partial \boldsymbol{\phi}_{1}}(\boldsymbol{\phi}, \boldsymbol{M}) \mid \frac{\partial \Psi_{n}}{\partial \boldsymbol{\phi}_{2}}(\boldsymbol{\phi}, \boldsymbol{M})\right\rangle
$$

provided all denominators in Eq. (2.2b) are nonvanishing in a sufficiently small open set containing $\boldsymbol{\phi}$ and $\boldsymbol{M}$. According to Eq. (2.2c), the Hall conductivity (2.2a) is now explicitly solely dependent on the linearly independent GSs.

In Ref. [6], it is argued that the equality

$$
\lim _{L_{1}, L_{2} \rightarrow \infty} \sigma_{\mathrm{H}}\left(\boldsymbol{\phi}_{0}, \boldsymbol{M}\right)=\lim _{L_{1}, L_{2} \rightarrow \infty} \int_{0}^{2 \pi} \int_{0}^{2 \pi} \frac{d \phi_{1} d \phi_{2}}{4 \pi^{2}} \sigma_{H}(\boldsymbol{\phi}, \boldsymbol{M})
$$

must necessarily hold for any arbitrarily chosen twisted boundary condition $\phi_{0}$ in the thermodynamic limit. Here, 


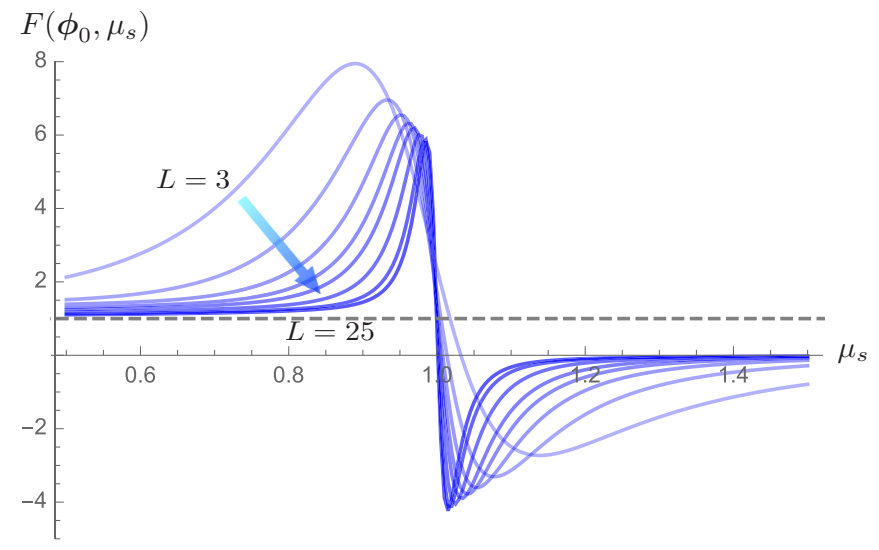

FIG. 1. Many-body Berry curvature $F\left(\phi_{0}, \mu_{\mathrm{s}}\right)$ for a noninteracting Chern insulator [specifically, the triangular lattice model defined by the noninteracting Hamiltonian (3.3)] for fixed value of flux $\boldsymbol{\phi}_{0}=(\pi-0.7,0)$, for a series of system sizes $L \equiv L_{1}=$ $L_{2}=3,5,7,9,11,15,21,25$. In the thermodynamic limit, the model has a topological phase transition in which the Chern number changes from 1 (gray line) to 0 at $\mu_{\mathrm{s}}=1$ (taking $t=1$ as energy unit). In the thermodynamic limit, $F\left(\phi_{0}, \mu_{\mathrm{s}}\right)$ becomes constant and thus independent of $\boldsymbol{\phi}_{0}$ and $\mu_{\mathrm{s}}$ except at the phase transition. The many-body Berry curvature $F\left(\boldsymbol{\phi}, \mu_{\mathrm{s}}\right)$ is singular at $\boldsymbol{\phi}_{\mathrm{c}}=(\pi, 0)$ and $\left(\mu_{\mathrm{s}} / t\right)_{\mathrm{c}}=1$ for $L \equiv L_{1}=L_{2}=3,5,7,9,11,15,21,25$.

the domain of integration is the torus defined by the twisted boundary conditions, to which we shall refer as the flux Brillouin zone (fBZ). The intuition for Eq. (2.3) is that the choice of the boundary conditions should not affect the values taken by the components of the conductivity tensor in an insulating phase after the thermodynamic limit has been taken.

We note that Eq. (2.3) implies that, as the thermodynamic limit is taken, the many-body Berry curvature $F_{n}(\boldsymbol{\phi}, \boldsymbol{M})$ becomes quantized and thus independent of $\boldsymbol{\phi}$ and $\boldsymbol{M}$, except for discontinuous changes at $\boldsymbol{M}$ that correspond to a topological phase transition at which the gap between the GS manifold and the excited states closes. If so, at least one denominator on the right-hand side of Eq. (2.2b) vanishes and the representation (2.2c) is ill defined. We illustrate this for a noninteracting lattice model in Fig. 1. This is distinct from the Berry curvature of single-particle bands, which generically has a momentum dependence even in the thermodynamic limit. Hence, scaling relations obeyed by $F(\boldsymbol{\phi}, \boldsymbol{M})$ as a function of $\boldsymbol{\phi}$ and $\boldsymbol{M}$, which we discuss in Sec. IV, can only be defined in systems of finite size.

Consequently, in the thermodynamic limit,

$$
\frac{\sigma_{\mathrm{H}}(\boldsymbol{M})}{\left(e^{2} / h\right)} \equiv \frac{1}{N_{g=1}} \sum_{n=1}^{N_{g=1}} C_{n}(\boldsymbol{M}),
$$

where

$$
C_{n}(\boldsymbol{M}) \equiv \lim _{L_{1}, L_{2} \rightarrow \infty} \int_{0}^{2 \pi} \int_{0}^{2 \pi} \frac{d \phi_{1} d \phi_{2}}{4 \pi^{2}} F_{n}(\boldsymbol{\phi}, \boldsymbol{M})
$$

is an integer-valued function of $\boldsymbol{M}$, called the Chern number of the $n$th state in the GS manifold. The Chern number $C_{n}(\boldsymbol{M})$ is a well-defined topological invariant of the bundle $\left\{\left|\Psi_{n}(\boldsymbol{\phi}, \boldsymbol{M})\right\rangle\right\}$ of many-body eigenstates over the fBZ, provided that the energy level that corresponds to $\left|\Psi_{n}(\boldsymbol{\phi}, \boldsymbol{M})\right\rangle$ does not cross any other level in the fBZ [6,10,11].

That the dimensionless quantum Hall conductivity on the left-hand side of Eq. (2.4a) must be a rational number in a FQH phase in the thermodynanic limit [12] is understood from the fact that the many-body Chern number (2.4b) is integer valued. Correspondingly, the right-hand side of Eq. (2.4a) is a noninteger rational number if and only if $N_{g=1}>1$ and the sum over all $C_{n}$ is not a multiple of $N_{g=1}$. Moreover, at any quantum phase transition driven by the parameters $\boldsymbol{M}$ by which the Hall conductivity changes discontinuously, the many-body Berry curvature (2.2b) must become singular somewhere in the fBZ. (This observation in the context of the quantum Hall and spin quantum Hall effect was made in Refs. [13] and [14], respectively. A similar argumentation can also be found in Refs. [15,16] for topological invariants in quantum spin chains.) This quantum phase transition is called a plateau transition to emphasize the fact that the quantum Hall conductivity is constant on either side of this transition. It is called a topological transition to emphasize that the phases on either side of this transition differ topologically through the values taken by the quantum Hall conductivity.

Away from the thermodynamic limit, i.e., when $L_{1}$ and $L_{2}$ are nonvanishing positive integers, the equality (2.4a) is no longer valid. Indeed, the Hall conductivity, defined through the Kubo formula for one choice of twisted boundary conditions, need not equal that for another choice of twisted boundary conditions away from the thermodynamic limit, contrary to what is implied by Eq. (2.4a) in the thermodynamic limit. The GS manifold, if degenerate in the thermodynamic limit, is generically nondegenerate away from the thermodynamic limit. On the other hand, the Chern number $(2.4 \mathrm{~b})$ remains quantized provided the energy eigenstate $\left|\Psi_{n}(\boldsymbol{\phi}, \boldsymbol{M})\right\rangle$ is nondegenerate away from the thermodynamic limit everywhere in the $\mathrm{fBZ}$ and in some region of parameter space.

In the following, we shall focus on the case where only one of the $N_{g=1}$ GSs in the thermodynamic limit has a nonvanishing Chern number,

$$
C_{n}(\boldsymbol{M})= \begin{cases}0, & \text { if } n \neq n^{\star}=1, \ldots, N_{g=1} \\ C_{\star}(\boldsymbol{M}) \neq 0, & \text { otherwise }\end{cases}
$$

deep in any insulating phase of parameter space. We have verified numerically that all cases studied in this paper fulfill the condition of Eq. (2.5) that only one state in the GS manifold has a nonvanishing Chern number, in agreement with previous analytical and numerical results $[11,17,18]$. In cases where more than one state in the GS manifold has a nonzero Chern number, then Weyl nodes will appear in a sequence of GS excited state level crossings. We shall not encounter such a case in this work. Now, even though a plateau transition is rounded by finite-size effects, the Chern number $C_{\star}(\boldsymbol{M})$ of the bundle of GSs $\left\{\left|\Psi_{n}(\boldsymbol{\phi}, \boldsymbol{M})\right\rangle\right\}$ over the fBZ is a discontinuous function of the parameters $\boldsymbol{M}$ that drive the plateau transition in the thermodynamic limit. Correspondingly, the Berry curvature $F_{\star}(\boldsymbol{\phi}, \boldsymbol{M})$ computed away from the thermodynamic limit must develop one or more singularities in the fBZ for critical values of $\boldsymbol{M}$ that drive the plateau transition in the thermodynamic limit as illustrated in Fig. 1. We shall further assume that the jump in $C_{\star}(\boldsymbol{M})$ results from a level touching between two many-body states $\left|\Psi_{1}(\boldsymbol{M})\right\rangle$ and $\left|\Psi_{2}(\boldsymbol{M})\right\rangle$, i.e., from one or 
more Weyl points (diabolical points) in $\boldsymbol{\phi}-\boldsymbol{M}$ space alluded to in Sec. I. In this situation, the Kubo formula (2.2c) contains a resonant denominator due to the two states participating in the level crossing. This yields the dominant contribution to the Berry curvature. This mechanism indeed describes all our exact diagonalization calculations. What will not be done in this paper is a finite-size scaling analysis to show that this assumption remains valid as the thermodynamic limit is approached. Below we reserve the notation $C(\boldsymbol{M}) \equiv C_{\star}(\boldsymbol{M})$ for the single nonzero Chern number in the GS manifold.

\section{FCI-TO-TRIVIAL TRANSITIONS}

\section{A. Models}

We focus on three widely used models of a single species of interacting hard-core particles (fermions or bosons) hopping on two-dimensional lattices made of $L_{1} \times L_{2}$ unit cells, with two sites per unit cell. Their general form is

$$
\widehat{H}:=\widehat{H}_{\text {kin }}+\widehat{H}_{\text {int }} \cdot
$$

The kinetic energy $\widehat{H}_{\text {kin }}$ may be written in reciprocal space as

$$
\widehat{H}_{\text {kin }}:=\sum_{k \in \mathrm{BZ}} \widehat{\Psi}_{k}^{\dagger} \mathcal{H}_{k} \widehat{\Psi}_{k},
$$

where $\widehat{\Psi}_{\boldsymbol{k}}^{\dagger} \equiv\left(\widehat{c}_{\boldsymbol{k}, A}^{\dagger}, \widehat{c}_{\boldsymbol{k}, B}^{\dagger}\right)$ is the spinor whose two components are made up from two single-particle creation operators with wave number $\boldsymbol{k}$ in the first Brillouin zone of crystal momenta (not to be confused with the fBZ of twists in the boundary conditions) and sublattice indices $A$ and $B$, respectively. The single-particle $2 \times 2$ matrix $\mathcal{H}_{\boldsymbol{k}}$ is here defined by

$$
\mathcal{H}_{\boldsymbol{k}}:=d_{0, \boldsymbol{k}} \tau_{0}+\boldsymbol{d}_{\boldsymbol{k}} \cdot \boldsymbol{\tau}+\mu_{\mathrm{s}} \tau_{3},
$$

where $\tau_{0}$ and $\boldsymbol{\tau}=\left(\tau_{1}, \tau_{2}, \tau_{3}\right)$ are the $2 \times 2$ unit and Pauli matrices in sublattice space, respectively, and $\mu_{\mathrm{s}}$ is a chemicalpotential imbalance between the two sublattices. For both fermions and bosons, the interaction $\widehat{H}_{\text {int }}$ is defined to be

$$
\widehat{H}_{\mathrm{int}}:=V_{1} \sum_{\langle\boldsymbol{i}, \boldsymbol{j}\rangle} \widehat{n}_{\boldsymbol{i}} \widehat{n}_{\boldsymbol{j}}+V_{2} \sum_{\langle\langle\boldsymbol{i}, \boldsymbol{j}\rangle\rangle} \widehat{n}_{\boldsymbol{i}} \widehat{n}_{\boldsymbol{j}},
$$

where $\widehat{n}_{i}:=\widehat{c}_{i}^{\dagger} \widehat{c}_{i}$ is the quasiparticle number operator on site $i$ of the lattice and $\langle\ldots\rangle$ and $\langle\langle\ldots\rangle\rangle$ denote nearest and nextnearest neighbors, respectively. The sign of the parameters $V_{1} \geqslant 0$ and $V_{2} \geqslant 0$ correspond to repulsive interactions.

The triangular-lattice model of Refs. [18-21] is defined by choosing $d_{0, \boldsymbol{k}}$ and $\boldsymbol{d}_{\boldsymbol{k}} \equiv\left(d_{1, \boldsymbol{k}}, d_{2, \boldsymbol{k}}, d_{3, \boldsymbol{k}}\right)^{\top}$ as

$$
\begin{aligned}
& d_{0, \boldsymbol{k}}:=2 t_{3} \sum_{j=1}^{3} \cos \left(2 \boldsymbol{k} \cdot \boldsymbol{\delta}_{j}\right), \\
& d_{j, \boldsymbol{k}}:=2 t \cos \left(\boldsymbol{k} \cdot \boldsymbol{\delta}_{j}\right), \quad j=1,2,3
\end{aligned}
$$

where $\boldsymbol{\delta}_{1}=(1 / 2,+\sqrt{3} / 2)^{\top}, \boldsymbol{\delta}_{2}=(1 / 2,-\sqrt{3} / 2)^{\top}$, and $\boldsymbol{\delta}_{3}=$ $-\left(\delta_{1}+\delta_{2}\right)$ [see Fig. 2(a)]. In all calculations for this triangularlattice model, we choose $t>0$ and $V_{2}=\mu_{\mathrm{s}}=0$. The $V_{1}-t_{3}$ phase diagram of this model at density $\rho=\frac{1}{3}$ particles per site has been mapped out in detail in Refs. $[18,21]$ and contains the competition between a FCI and a charge-density wave (CDW) state.
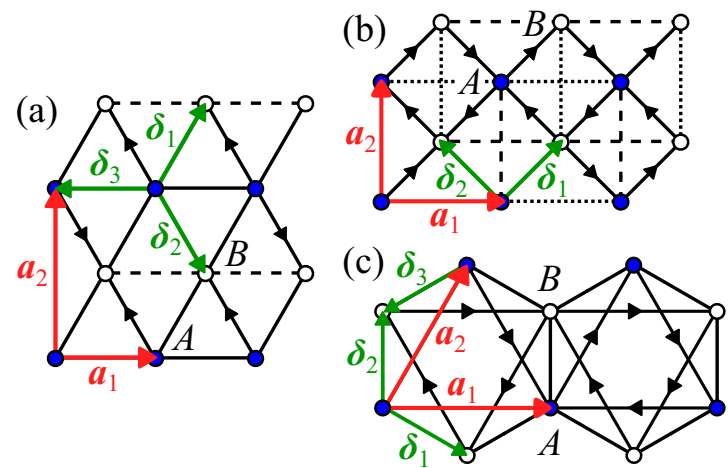

FIG. 2. Schematic definition of (a) triangular-lattice model of Eq. (3.3), (b) checkerboard-lattice model of Eq. (3.4), and (c) honeycomb-lattice model of Eq. (3.5). Hoppings in the direction of an arrow add $\varphi$ to the phase of the electron wave function, hopping in the opposite direction subtracts $\varphi(\varphi=\pi / 2$ for triangular-lattice model, $\varphi=\pi / 4$ for checkerboard-lattice model, $\varphi=0.4 \pi$ for honeycomblattice model); dashed (dotted) lines denote hoppings with a negative (positive) sign; in all models, third-neighbor hoppings are uniform and are omitted for clarity.

The model of Refs. [8,22] is defined on the checkerboard lattice with primitive vectors $\boldsymbol{a}_{1}=(1,0)^{\top}$ and $\boldsymbol{a}_{2}=(0,1)^{\top}$ as

$$
\begin{aligned}
d_{0, \boldsymbol{k}} & :=2 t_{3}\left(\cos 2 \boldsymbol{k} \cdot \boldsymbol{\delta}_{1}+\cos 2 \boldsymbol{k} \cdot \boldsymbol{\delta}_{2}\right), \\
d_{1, \boldsymbol{k}} & :=2 t \cos \varphi\left(\cos \boldsymbol{k} \cdot \boldsymbol{\delta}_{1}+\cos \boldsymbol{k} \cdot \boldsymbol{\delta}_{2}\right), \\
d_{2, \boldsymbol{k}} & :=2 t \sin \varphi\left(\cos \boldsymbol{k} \cdot \boldsymbol{\delta}_{1}+\cos \boldsymbol{k} \cdot \boldsymbol{\delta}_{2}\right), \\
d_{3, \boldsymbol{k}} & :=2 t_{2}\left(\cos \boldsymbol{k} \cdot \boldsymbol{a}_{1}-\cos \boldsymbol{k} \cdot \boldsymbol{a}_{2}\right),
\end{aligned}
$$

where $t, t_{2}$, and $t_{3}$ are nearest-, second-nearest-, and thirdnearest-neighbor hopping amplitudes, respectively, and $\boldsymbol{\delta}_{1}=$ $(\sqrt{2} / 2, \sqrt{2} / 2)^{\top}, \boldsymbol{\delta}_{2}=(-\sqrt{2} / 2, \sqrt{2} / 2)^{\top}$ [see Fig. 2(b)]. In the following, we fix $t<0, t_{2} / t=-1 /(2+\sqrt{2}), t_{3} / t=$ $-1 /(2+2 \sqrt{2})$, and $\varphi=\pi / 4$, which for $V_{1}=V_{2}=0$ generates a nearly flat, topologically nontrivial lower band with a nonzero Chern number [22]. The interacting model at density $\rho=\frac{1}{6}$ particles per site (filling $v=\frac{1}{3}$ of the lower band in the noninteracting limit) has been studied extensively $[8,20,23-25]$. In particular, it was found that (a) the GS of the model is a topologically ordered FCI for $V_{2}=\mu_{\mathrm{s}}=0$ and $V_{1}$ ranging from moderate to infinite $[20,23]$, (b) a large enough $V_{2}$ drives the model into a Fermi-liquid-like phase for any $V_{1}>0$ [23], and (c) for large enough $\mu_{\mathrm{s}}$ the model transitions into a charge-modulated, topologically trivial state $[8,20]$. Our results are fully consistent with these findings.

Finally, we define the Haldane model on the honeycomb lattice as $[8,26]$

$$
\begin{aligned}
& d_{0, \boldsymbol{k}}:=2 t_{2} \cos \varphi \sum_{i=1}^{3} \cos \boldsymbol{k} \cdot \boldsymbol{a}_{i}, \\
& d_{1, \boldsymbol{k}}:=t \sum_{i=1}^{3} \cos \boldsymbol{k} \cdot \boldsymbol{\delta}_{i},
\end{aligned}
$$




$$
\begin{aligned}
d_{2, \boldsymbol{k}}:= & t \sum_{i=1}^{3} \sin \boldsymbol{k} \cdot \boldsymbol{\delta}_{i}, \\
d_{3, \boldsymbol{k}}:= & -2 t_{2} \sin \varphi \sum_{i=1}^{3} \sin \boldsymbol{k} \cdot \boldsymbol{a}_{i} \\
& +t_{3}\left[e^{i \boldsymbol{k} \cdot\left(\boldsymbol{\delta}_{2}+\boldsymbol{a}_{1}\right)}+e^{i \boldsymbol{k} \cdot\left(\boldsymbol{\delta}_{2}-\boldsymbol{a}_{1}\right)}+e^{-2 i \boldsymbol{k} \cdot \boldsymbol{\delta}_{2}}\right],
\end{aligned}
$$

with $\boldsymbol{a}_{1}=(\sqrt{3}, 0)^{\top}, \boldsymbol{a}_{2}=(\sqrt{3} / 2,3 / 2)^{\top}, \boldsymbol{a}_{3}=\boldsymbol{a}_{2}-\boldsymbol{a}_{1}, \boldsymbol{\delta}_{1}=$ $(\sqrt{3} / 2,-1 / 2)^{\top}, \quad \delta_{2}=(0,1)^{\top}$, and $\delta_{3}=(-\sqrt{3} / 2,-1 / 2)^{\top}$ [see Fig. 2(c)]. Following Ref. [27], we set $t_{2} / t=0.60$, $t_{3} / t=0.58, \mu_{s}=0$, and $\varphi=0.4 \pi$. The bosonic version of this model has a $v=\frac{1}{2}$ FCI GS for any $V_{1} \geqslant 0$, but transitions to a CDW when $V_{2}>V_{1}$ [27].

The ranges of parameters for all three models are chosen so as to easily identify and characterize quantum phase transitions. As long as pointlike degeneracies (nodal points) appear in the fBZ for a critical value of some parameter, then our methodology is applicable and small changes in model parameters are inconsequential.

The many-body Berry curvature can be evaluated accurately for finite clusters using the Lanczos method [18-21]. In the examples studied in this work, we study the topological phase transition between a degenerate FCI state, with an accurately quantized topological invariant $C \neq 0$, and a topologically trivial state with $C=0$. Depending on geometrical details, the FCI GSs may or may not all be in the same symmetry sector [9]. In the former case, there is an energy splitting between quasidegenerate GSs of the FCI phase in finite-size numerics and it is expected that only one of the GSs that we shall label by $n=n_{\star}$ will carry the entire topological response $[11,17]$. Here, we focus on this case and therefore all the results we present are for the symmetry sector that contains the GSs. In the $v=\frac{2}{3}$ FCI phase of the fermionic triangular-lattice model, one of the three quasidegenerate/finite-size split FCI states has $C_{\star}=2$, whereas the other two have $C=0$ (average $C$ is $\frac{2}{3}$, as expected for a fractional quantum Hall state at this filling), while in the $v=\frac{1}{3}$ FCI phase of the fermionic checkerboard-lattice model one GS has $C_{\star}=1$ and the other two have $C=0$ (average $C$ is $\frac{1}{3}$ ). The GS of the bosonic Haldane model at $\rho=\frac{1}{4}$ is a twice-(quasi-)degenerate $v=\frac{1}{2}$ FCI state, with one of the two states in the GS manifold having $C_{\star}=1$ and the other $C=0$ (average $C$ is $\frac{1}{2}$ ). For all models, we investigate a topological phase transition out of the FCI phase upon variation of parameters; $V_{1}$ and $t_{3}$ in the triangular-lattice model, $V_{2}$ and $\mu_{\mathrm{s}}$ in the checkerboard-lattice model, and $V_{2}$ in the Haldane model. We have evaluated $C$ on both sides of the transitions by performing the integration in Eq. (2.4b) numerically on finite grids of the fBZ of sizes up to $64 \times 64$ and have obtained accurately quantized values, in agreement with the expected behavior.

\section{B. Nodes between many-body energy levels}

The generalization of nodal points found in noninteracting band structures to interacting systems occurs when the manybody gap closes at isolated points in the fBZ. As we detail in Appendix D, spatial symmetries of a system with periodic boundary conditions translate to symmetries in the fBZ when twisted boundary conditions are imposed. The effect of symmetries on the energy eigenvalue spectrum in the fBZ has also been discussed in Ref. [13]. There, it is argued that the Chern number (2.5) for a many-body interacting Hamiltonian with inversion symmetry can only change by an odd integer if the locations in the $\mathrm{fBZ}$ at which the level crossing takes place is an inversion-symmetric point. We shall encounter other symmetries that constrain the points in the fBZ at which diabolical points must occur. For instance, it was shown in Ref. [28] that Weyl nodes on a point in momentum space whose little group contains a fourfold or sixfold rotation can be of higher charge. Similarly, if an interacting many-body system has a threefold rotation symmetry, band touchings at high-symmetry points in the fBZ may be of higher charge. We will discuss one such example below.

First, we focus on the critical point between $v=\frac{2}{3} \mathrm{FCI}$ and CDW phases of the fermionic triangular-lattice model of Eq. (3.3) which is a singly charged Weyl node. In this example, upon fine tuning in parameter space to criticality, the pair of many-body energy dispersions in the fBZ for the many-body state supporting the many-body Chern number $C_{\star}=2$, on the one hand, and that for the many-body state supporting a $\mathrm{CDW}$, on the other hand, touch at the two inequivalent points $\boldsymbol{\phi} \equiv\left(\phi_{1}, \phi_{2}\right)=(0,0)$ and $\boldsymbol{\phi}=(0, \pi)$. Figure 3 demonstrates this level touching. Figure 3 also displays the dependence of the energy dispersion of these many-body states on the twisted boundary conditions. We note that (i) the dispersion in the $\mathrm{fBZ}$ of this pair of many-body states is linear around the nodal points, (ii) the Berry curvatures of this pair of many-body states have the same sign at both points in the fBZ, and (iii) the presence of two points in the fBZ at which the Berry curvature diverges leads to a jump of magnitude 2 in the Chern numbers of the two states involved in the transition. The transition in the fermionic checkerboard-lattice model of Eqs. (3.4) has the same features, with the only difference that for this model there is only one nonequivalent nodal point, namely, at the center $\boldsymbol{\phi}=(0,0)$ of the fBZ, and the corresponding jump in the magnitude of the Chern number of the GS manifold upon crossing the quantum critical point in parameter space is thus 1 .

The bosonic interacting Haldane model at density $\rho=\frac{1}{4}$ (number of particles divided by the number of sites) exhibits an instance of higher-order touching of many-body energies in the fBZ when crossing in parameter space through the transition from a $v=\frac{1}{2}$ bosonic FCI GS manifold to a charge-ordered GS manifold upon increasing second-neighbor repulsion $V_{2}$ [27]. The phenomenology at this transition as a function of increasing $V_{2}$ in parameter space is the following. (1) For small $V_{2} \lesssim 1.446$ the FCI and CDW many-body energy levels are fully separated in energy, with the $C_{\star}=1$ FCI many-body state being higher in energy than its quasidegenerate counterpart (which has $C=0$ ) and lower in energy than the CDW many-body state. The many-body Berry curvatures are smooth functions everywhere in the fBZ. (2) When $V_{2}$ reaches the lower critical value $V_{2} \sim 1.446$, the many-body $C_{\star}=1 \mathrm{FCI}$ and CDW dispersions in the fBZ touch at $\phi \equiv\left(\phi_{1}, \phi_{2}\right)=(0,0)$, without a concomitant divergence of the many-body Berry curvature at that point, and disperse quadratically with the deviations $\phi_{1}$ and $\phi_{2}$ away from $\phi_{1}=0$ and $\phi_{2}=0$ as shown in Fig. 4. (3) For $1.446 \lesssim V_{2} \lesssim 1.642$ the two many-body levels near avoid one another, evidently due to many-body level 
(a) nontrivial: $V_{1} / t=1.11, C=2$
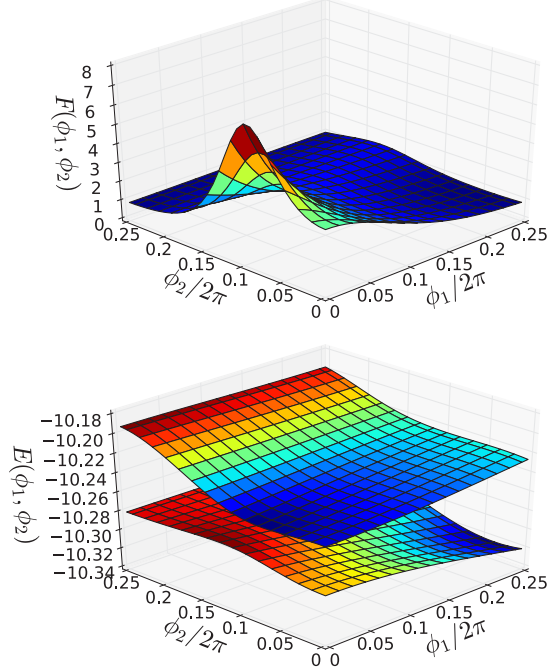

(b) critical: $V_{1} / t \sim 1.23$
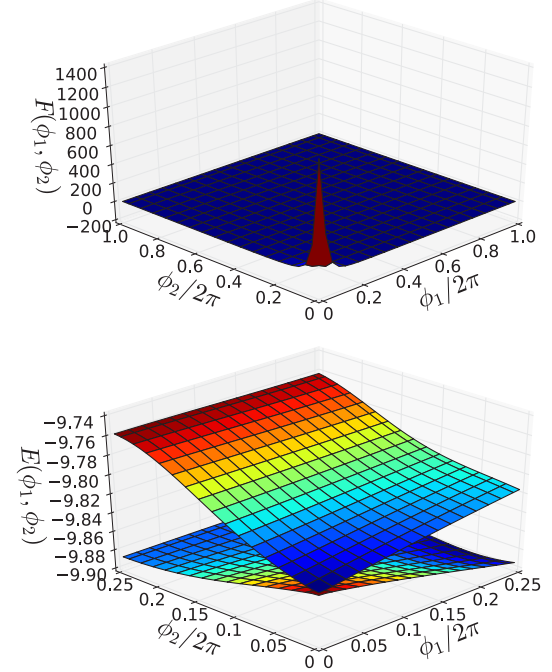

(c) trivial: $V_{1} / t=1.3, C=0$
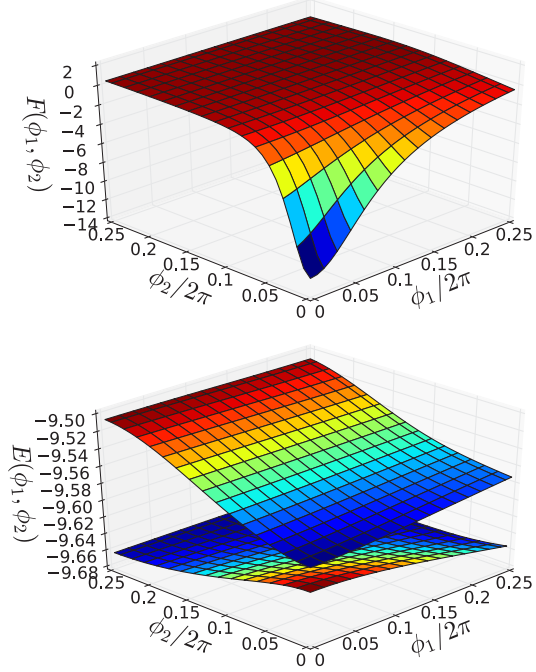

FIG. 3. Many-body Berry curvatures (top panels) and many-body energy dispersions in the fBZ (bottom panels) for the FCI and CDW states of the fermionic model defined by Eqs. (3.3) on a triangular lattice made of 18 sites at the fermionic density (number of fermions divided by the number of sites) $\rho=\frac{1}{3}$ holding $t_{3} / t=0.22$ fixed. In (a), $V_{1} / t=1.11$ (topologically nontrivial $v=\frac{2}{3}$ FCI phase). In (b), $V_{1} / t=1.23$ (close to the transition point). In (c), $V_{1} / t=1.3$ (topologically trivial CDW phase).

repulsion, at three points in the $\mathrm{fBZ}$ that move away from $\phi=$ $(0,0)$ along high-symmetry lines upon increasing $V_{2}$, while the many-body Berry curvature develops three corresponding maxima at the same points (see Fig. 5). (4) When $V_{2}$ reaches the upper critical value $V_{2} \sim 1.642$, the previously avoided many-body crossings reach the points $\phi=(\pi, 0), \phi=(0, \pi)$, and $\boldsymbol{\phi}=(\pi, \pi)$, at which the many-body gap now vanishes exactly and the many-body Berry curvature diverges. (5) For $V_{2} \gtrsim 1.642$ the many-body spectrum becomes gapped again, with the many-body $C_{\star}=1 \mathrm{FCI}$ and $C=0 \mathrm{CDW}$ levels having exchanged places along the many-body energy axis.

Although the phase encountered for $1.446 \lesssim V_{2} \lesssim 1.642$ is characterized by avoided many-body level crossings, the Chern numbers of the corresponding many-body states are nonetheless well defined since the energy spectrum is actually
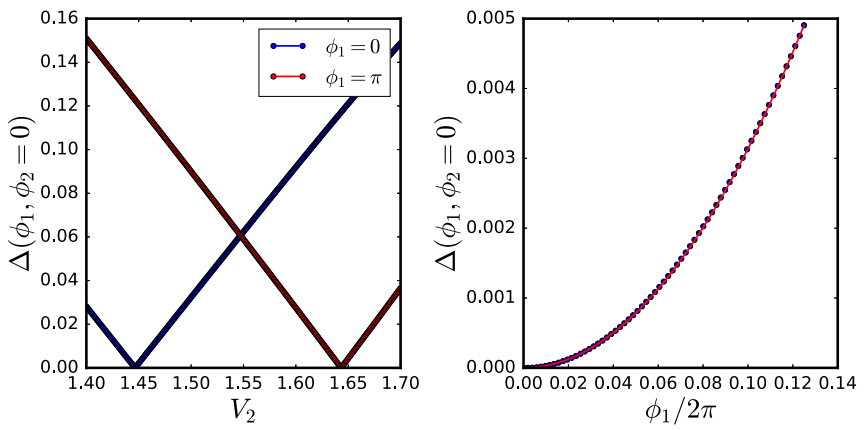

FIG. 4. Many-body energy gaps between the FCI and CDW energy levels for the bosonic interacting Haldane model of Ref. [27] on a honeycomb lattice made of 32 sites, as defined in Eq. (3.5). The average density of hard-core bosons per site is $\rho=\frac{1}{4}$ and $V_{1}=0$. In (a), $V_{2}$ is varied holding $\phi \equiv\left(\phi_{1}, \phi_{2}\right)$ fixed to either $\phi=(0,0)$ or $\boldsymbol{\phi}=(\pi, 0)$. (a) Shows that the many-body gap closes at $V_{2} \sim 1.446$ and 1.642, respectively. In (b), $\phi_{1}$ is varied holding $V_{2}=1.446$ and $\phi_{2}=0$ fixed. The red line in (b) is the fit $\beta_{1} \phi_{1}^{2}$ with $\beta_{1} \simeq \pi / 10$. gapped, albeit by finite-size effects (see Fig. 5). For $V_{2}<$ 1.446, we find the many-body Chern numbers of the FCI and CDW states to be 1 and 0 , respectively. For $1.446 \lesssim V_{2} \lesssim$ 1.642 , the many-body Chern numbers of the "hybridized" states, whose levels repel one another and are separated by the finite-size gap, jump to the values 3 and -2 , respectively. This behavior has been previously observed for avoided crossings in a disordered FCI model [18] and indicates that the Chern number is mathematically well defined in finite clusters with (a)

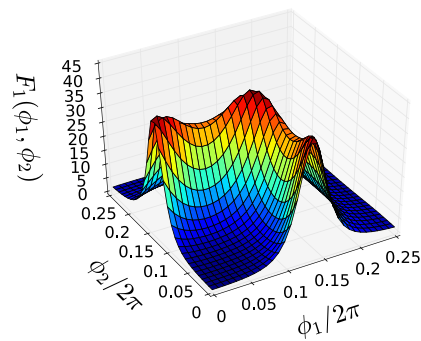

(c)

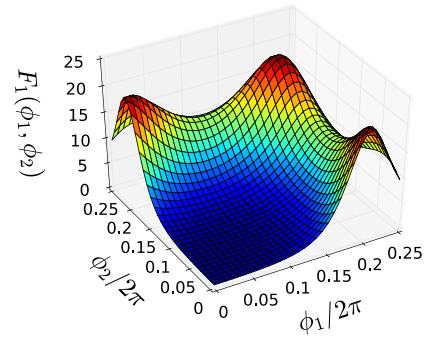

(b)

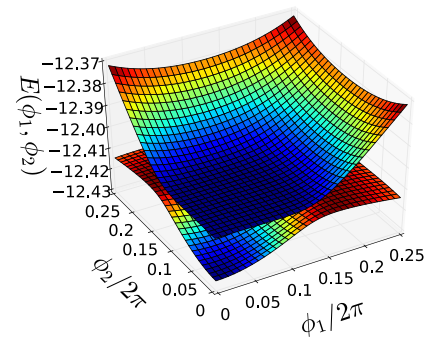

(d)

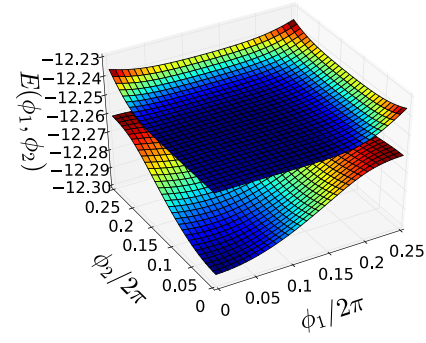

FIG. 5. (a), (c) Berry curvature of the state $\left|\Psi_{1}\right\rangle$ corresponding to first-excited energy level $E_{1}$ and (b), (d) energy levels $E_{1}$ and $E_{2}$ in flux space for a 32-site cluster of the interacting Haldane model of Ref. [27], as defined in Eq. (3.5), at density $\rho=\frac{1}{4}$ hard-core bosons per site with $V_{1}=0$ and (a), (b) $V_{2} / t=1.48$, which is close to the critical point, and (c), (d) $V_{2} / t=1.52$ that is far away from it. 
finite-size gaps, even though the energy spectrum is expected to become gapless in the thermodynamic limit. Note that the first transition at $V_{2} \sim 1.446$ is accompanied by quadratically dispersing many-body energy levels around a single nodal point from the fBZ and a jump of magnitude 2 in the many-body Chern numbers, whereas the second transition at $V_{2} \sim 1.642$ is accompanied by linearly dispersing many-body energy levels around three nodal points from the fBZ and a jump of magnitude 3 in many-body Chern numbers.

\section{Phenomenological classification by dispersion in the $\mathrm{fBZ}$}

The cases detailed above suggest analogies between the interacting systems studied here and Weyl semimetals. In the above examples, the many-body Berry curvature of a state is fully parametrized by a vector $\phi$ of boundary twists and a vector $\boldsymbol{M}$ of parameters as $F_{n}(\boldsymbol{\phi}, \boldsymbol{M})$. This many-body Berry curvature integrates to a quantized Chern number in the $\phi$ plane for $\boldsymbol{M} \neq \boldsymbol{M}_{\mathrm{c}}$. Finally, there is a jump in the many-body Chern number that depends on the number and order of level touchings that occur at $\boldsymbol{M}=\boldsymbol{M}_{\mathrm{c}}$.

In Weyl semimetals, the single-particle Berry curvature is fully parametrized by a vector of "in-plane" momenta $\boldsymbol{k}_{\|}$and an "out-of-plane" momentum $k_{\perp}$ as $F_{n}\left(\boldsymbol{k}_{\|}, k_{\perp}\right)$. This single-particle Berry curvature integrates to a quantized Chern number in the $\boldsymbol{k}_{\|}$plane for $k_{\perp} \neq k_{\perp, \mathrm{c}}$. Finally, there is a jump in the single-particle Chern number that depends on the number and order of level touchings (Weyl nodes) that occur across $k_{\perp} \neq k_{\perp, \mathrm{c}}$.

It is known that higher-order Weyl nodes are only possible in the presence of additional point-group symmetries and split into single Weyl nodes when the symmetry protecting them is broken [27,28]. In an analogous fashion, we observe that the quadratic nodal point appearing at $\phi=(0,0)$ in the interacting Haldane model splits into two linear nodes upon lowering the rotational symmetry by introducing a small mass imbalance between sublattices $A$ and $B$.

In noninteracting systems, the presence of nodes in a band structure at isolated points in $\boldsymbol{k}$ space can be $a$ priori determined via symmetry arguments [29]. Whether the manybody energy landscape in $\phi$ space of an arbitrary interacting system contains nodal points or not is less straightforward to determine. Even though, in this work, our approach to answering this question has been trial and error, some arguments hold generally. Gaplessness can appear at high-symmetry points (HSPs) in the fBZ associated with space-group symmetries, or in the form of accidental band crossings at arbitrary points in the fBZ [11,30,31]. However, any degeneracies away from HSPs need to also obey all space-group symmetries, which means they must come in multiples. For example, if a degeneracy occurs at an arbitrary (non-high-symmetry) point in the fBZ of a system with $C_{6}$ symmetry, for instance, then there necessarily need to be five more copies of this degeneracy [13]. Furthermore, symmetry considerations do not preclude an exact degeneracy of many-body levels leading to a divergence of the Berry curvature on a one-dimensional manifold in the fBZ, with a $\boldsymbol{\phi}$-space dispersion resemblant of noninteracting nodal-line semimetals. We shall not address this case here.
For all the three models studied in Sec. III B, near the critical point $\boldsymbol{M}_{\mathrm{c}}$ and close to the nodal point $\boldsymbol{\phi}_{\mathrm{c}}$, the many-body gap between the many-body energy levels $E_{1}(\boldsymbol{\phi}, \boldsymbol{M})$ and $E_{2}(\boldsymbol{\phi}, \boldsymbol{M})$ corresponding to topologically nontrivial and trivial manybody states can be heuristically fitted by the simple form

$$
\left(E_{2}-E_{1}\right)(\boldsymbol{\phi}, \boldsymbol{M}) \approx \sqrt{\beta_{1}\left(\delta \phi_{1}\right)^{2 p}+\beta_{2}\left(\delta \phi_{2}\right)^{2 p}+\beta_{3}\left|\boldsymbol{M}-\boldsymbol{M}_{\mathrm{c}}^{\star}\right|^{2}},
$$

where $\boldsymbol{M}_{\mathrm{c}}^{\star}$ is the point on the $(m-1)$-dimensional phase boundary that is closest to $\boldsymbol{M}$ and where $\delta \phi_{1}$ and $\delta \phi_{2}$ are sufficiently small coordinates measured relative to $\phi_{\mathrm{c}} \equiv$ $\left(\phi_{\mathrm{c} 1}, \phi_{\mathrm{c} 2}\right)^{\top}$, while $\beta_{1}, \beta_{2}$, and $\beta_{3}$ are fitting parameters, and $\boldsymbol{M}$ is sufficiently close to $\boldsymbol{M}_{\mathrm{c}}^{\star}$. For all the nodal points we have encountered in our calculations, the power $p_{d}$ is always found to be an integer, and it is equal to 1 for divergences at HSPs in the triangular and the checkerboard models, and 2 (1) for the critical point at $V_{2} \sim 1.446\left(V_{2} \sim 1.642\right)$ in the honeycomb model. By monitoring numerically the Chern number $C$ of the state corresponding to the lower of the two levels as the system undergoes the level crossing upon variation of $\boldsymbol{M}$, we find that in all these cases the jump $\Delta C$ in the Chern number obeys the empirical relation

$$
\Delta C=\sum_{d=1}^{N_{\mathrm{div}}} p_{d}
$$

where $N_{\text {div }}$ denotes the number of nodes that develop at points $\boldsymbol{\phi}_{\mathrm{c}, d}$, with $d=1, \ldots, N_{\mathrm{div}}$, in the fBZ as one approaches a critical point, and $p_{d}$ is the corresponding integer governing the dispersion in $\phi$ close to the $d$ th HSP. Interestingly, this empirical result mirrors what happens in noninteracting systems, where Eqs. (3.6) and (3.7) can be rigorously related to one another $[27,28,32,33]$. In all the transitions we have investigated, the Berry curvature always has the same sign at all the HSPs of the fBZ where it becomes divergent simultaneously.

\section{BERRY CURVATURE RENORMALIZATION GROUP}

The similarities that we have observed in Sec. III when comparing the many-body Berry curvatures to the singleparticle Berry curvatures of noninteracting semimetals close to Weyl nodes motivate us to develop a scaling procedure for the many-body Berry curvature that is the many-body counterpart to the one applied to the single-particle Berry curvature in Refs. [33-35]. We then apply this scaling procedure to the FCI-to-trivial transitions presented in Sec. III. We detail the protocol below, which we refer to as Berry curvature renormalization group (BCRG), for the two scenarios $p_{d}=1$ and 2 uncovered in Sec. III.

The procedure we introduce relies on positing the functional form for the singularity of the Berry curvature $F_{\star}(\boldsymbol{\phi}, \boldsymbol{M})$ and the applicability of a scaling relation that ties the dependence of $F_{\star}(\boldsymbol{\phi}, \boldsymbol{M})$ on $\boldsymbol{\phi}$ to that on the parameters $\boldsymbol{M}$ that drive the plateau transition in the thermodynamic limit. [The index $\star$ in $F_{\star}(\boldsymbol{\phi}, \boldsymbol{M})$ was defined below Eq. (2.5).] Our approach is phenomenological in that we do not justify the validity of these assumptions on the basis of general principles. Instead, we 
confirm the fulfillment of these conditions on a case-by-case basis with exact diagonalization.

\section{A. Strategy}

The BCRG approach relies on the following three assumptions.

Assumption 1. The many-body Berry curvature is an even function of $\boldsymbol{\phi}$ around all the high-symmetry points (HSPs) defined by $\boldsymbol{\phi}_{\mathrm{c}}=-\boldsymbol{\phi}_{\mathrm{c}}$ modulo $\left(2 \pi n_{1}, 2 \pi n_{2}\right)$ for any pair of integers $n_{1}$ and $n_{2}$, i.e.,

$$
F_{\star}\left(\boldsymbol{\phi}_{\mathrm{c}}+\delta \boldsymbol{\phi}, \boldsymbol{M}\right)=F_{\star}\left(\boldsymbol{\phi}_{\mathrm{c}}-\delta \boldsymbol{\phi}, \boldsymbol{M}\right),
$$

where the Berry curvature $F_{\star}\left(\boldsymbol{\phi}_{\mathrm{c}}, \boldsymbol{M}\right)$ was defined implicitly by Eq. (2.5).

If there exists a quantum critical point $\boldsymbol{M}_{\mathrm{c}}$ across which the quantum Hall conductivity (2.4a) changes discontinuously, we then posit either one of the following two scenarios upon approaching in parameter space the quantum critical point $\boldsymbol{M}_{\mathrm{c}}$.

Assumption 2.1. The Berry curvature (2.2b) displays a local extremum at $\boldsymbol{\phi}_{\mathrm{c}}$ as a function of $\boldsymbol{\phi}$, holding $\boldsymbol{M}$ fixed and close to $\boldsymbol{M}_{\mathrm{c}}$. This extremum changes from being a local maximum to a local minimum as $\boldsymbol{M}$ is varied across $\boldsymbol{M}_{\mathrm{c}}$. The Berry curvature $F_{\star}\left(\boldsymbol{\phi}_{\mathrm{c}}, \boldsymbol{M}\right)$ diverges as $\boldsymbol{M} \rightarrow \boldsymbol{M}_{\mathrm{c}}$. For example, the dependence on $\delta \boldsymbol{\phi}$ given by the Ornstein-Zernike (OZ) scaling function

$$
F_{\mathrm{OZ}}\left(\boldsymbol{\phi}_{\mathrm{c}}+\delta \boldsymbol{\phi}, \boldsymbol{M}\right):=\frac{F_{\star}\left(\boldsymbol{\phi}_{\mathrm{c}}, \boldsymbol{M}\right)}{1+\xi_{1}^{2}(\boldsymbol{M}) \delta \phi_{1}^{2}+\xi_{2}^{2}(\boldsymbol{M}) \delta \phi_{2}^{2}},
$$

for some dimensionless functions $\xi_{1}(\boldsymbol{M})$ and $\xi_{2}(\boldsymbol{M})$, satisfies this assumption. It then follows that both $\xi_{1}^{2}(\boldsymbol{M})$ and $\xi_{2}^{2}(\boldsymbol{M})$ must share the same singular behavior with the Berry curvature (2.2b) when $\boldsymbol{\phi}$ is held fixed at $\boldsymbol{\phi}_{\mathrm{c}}$ while $\boldsymbol{M} \rightarrow \boldsymbol{M}_{\mathrm{c}}$. We shall subsequently call this assumption the peak-divergence scenario.

Assumption 2.2. The Berry curvature (2.2b) has a continuous mountain ridge (valley) that surrounds $\boldsymbol{\phi}_{\mathrm{c}}$ as a function of $\boldsymbol{\phi}$, holding $\boldsymbol{M}$ fixed and close to $\boldsymbol{M}_{\mathrm{c}}$. These extrema change from being local maxima to local minima as $\boldsymbol{M}$ is varied across $\boldsymbol{M}_{\mathrm{c}}$. These extrema collapse to $\boldsymbol{\phi}_{\mathrm{c}}$ and the Berry curvature at any point along these extrema diverges as $\boldsymbol{M} \rightarrow \boldsymbol{M}_{\mathrm{c}}$. We shall subsequently call this assumption the ring-divergence scenario.

Assumption 3. For any $\delta \boldsymbol{\phi} \equiv|\delta \boldsymbol{\phi}| \widehat{\delta \phi}$ nonvanishing yet not too large, there exists one and only one pair $\delta \phi^{\prime} \equiv\left|\delta \boldsymbol{\phi}^{\prime}\right| \widehat{\delta \phi}$ and $\boldsymbol{M}^{\prime}$ such that

$$
F_{\star}\left(\boldsymbol{\phi}_{\mathrm{c}}+\delta \boldsymbol{\phi}, \boldsymbol{M}\right)=F_{\star}\left(\boldsymbol{\phi}_{\mathrm{c}}+\delta \boldsymbol{\phi}^{\prime}, \boldsymbol{M}^{\prime}\right) .
$$

Defining $\left|\delta \boldsymbol{\phi}^{\prime}\right| /|\delta \boldsymbol{\phi}| \equiv b$, Assumption 2.1 demands that $0<b<1$, whereby $F_{\star}\left(\boldsymbol{\phi}_{\mathrm{c}}, \boldsymbol{M}\right)$ is a local maximum as a function of $\boldsymbol{\phi}$ holding $\boldsymbol{M}$ fixed. Assumption 2.2 demands that $b>1$, whereby $F_{\star}\left(\boldsymbol{\phi}_{\mathrm{c}}+\delta \boldsymbol{\phi}, \boldsymbol{M}\right)$ is a mountain ridge (valley). The intuition for Eq. (4.3) is captured by Fig. 6. The scaling ansatz (4.3) defines a flow in parameter space from $\boldsymbol{M}$ to $\boldsymbol{M}^{\prime}$, along which the divergence of the Berry curvature is reduced, and hence the system is moving away from the critical point, as explained in Appendix A. This flow can be encoded into a differential equation as follows. We define the scaling direction to be $\widehat{\delta \boldsymbol{\phi}}$. We define the infinitesimal scaling coordinate to be $d \ell \equiv|\delta \boldsymbol{\phi}|^{2}$. We define the infinitesimal change in the
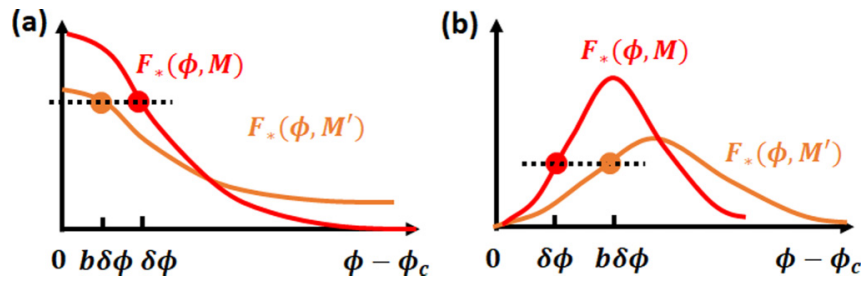

FIG. 6. (a), (b) Illustrate the scaling procedure for Assumptions 2.1 (peak-divergence scenario) and 2.2 (ring-divergence scenario), respectively, which demands the red dot to be equal to the orange dot to solve for the $\boldsymbol{M}^{\prime}$, as indicated by the dashed line. The profile of Berry curvature evolves from the red lines that have a large divergence to the orange lines that have a small divergence under this procedure, without changing the topological invariant, hence the system flows away from the critical point.

parameters to be $d \boldsymbol{M} \equiv \boldsymbol{M}^{\prime}-\boldsymbol{M}$. We do a Taylor expansion of Eq. (4.3) in powers of $\delta \boldsymbol{\phi}$ and $d \boldsymbol{M}$ and use the assumption (2) that $\nabla_{\phi} F_{\star}=0$ at $\phi_{\mathrm{c}}$ and $\boldsymbol{M}$. If so, there follows the partial differential equation

$$
\left.\left.\nabla_{\boldsymbol{M}} F_{\star}\right|_{\boldsymbol{\phi}_{\mathrm{c}}, \boldsymbol{M}} \cdot \frac{d \boldsymbol{M}}{d \ell}=\left(\frac{1-b^{2}}{2}\right) \widehat{\delta \boldsymbol{\phi}} \cdot \nabla_{\boldsymbol{\phi}}\right)\left.^{2} F_{\star}\right|_{\boldsymbol{\phi}_{\mathrm{c}}, \boldsymbol{M}}
$$

Assuming that $\left.\nabla_{\boldsymbol{M}} F_{\star}\right|_{\boldsymbol{\phi}_{c}, \boldsymbol{M}}$ is nonvanishing and performing this scaling procedure independently for each tuning parameter $M_{i}$, with $i=1, \ldots, m$, one can rearrange Eq. (4.4) to obtain a closed expression for $d M_{i} / d \ell$. The row vector $\left(d M_{1} / d \ell, \ldots, d M_{m} / d \ell,\right)^{\top} \equiv(d \boldsymbol{M} / d \ell)^{\top}$ delivers the BCRG flow in the $m$-dimensional parameter space. The critical points $\boldsymbol{M}_{\mathrm{c}}$ are identified numerically from the BCRG flow as the $(m-1)$-dimensional surface on which the flow directs away from the point at which the flow rate diverges. The fixed points $\boldsymbol{M}_{\mathrm{f}}$ are the points where the flows vanish. They can be either stable or unstable depending on the direction of the BCRG flow. In short,

$$
\begin{array}{r}
\text { Critical point: }\left|\frac{d \boldsymbol{M}}{d \ell}\right| \rightarrow \infty \text {, flow directs away, } \\
\text { Stable fixed point: }\left|\frac{d \boldsymbol{M}}{d \ell}\right| \rightarrow 0 \text {, flow directs into, } \\
\text { Unstable fixed point: }\left|\frac{d \boldsymbol{M}}{d \ell}\right| \rightarrow 0, \text { flow directs away. }
\end{array}
$$

To numerically estimate the partial differential equation (4.4), we use the triplet of values $F_{\star}\left(\boldsymbol{\phi}_{\mathrm{c}}, \boldsymbol{M}\right), F_{\star}\left(\boldsymbol{\phi}_{\mathrm{c}}+\Delta \phi_{i} \widehat{\delta \boldsymbol{\phi}}, \boldsymbol{M}\right)$, and $F_{\star}\left(\phi_{\mathrm{c}}, \boldsymbol{M}+\Delta M_{i} \widehat{\boldsymbol{M}}_{i}\right)$.

A couple of remarks are in order. First, this approach implicitly assumes that the bulk gap continuously reduces as $\boldsymbol{M}$ approaches $\boldsymbol{M}_{\mathrm{c}}$ and vanishes exactly at the critical point. This gap closing must be accompanied by the divergence of the many-body Berry curvature, as per Eq. (2.2b). This is not necessarily the case for an arbitrary topological phase transition. On the one hand, states whose levels cross with varying $\boldsymbol{M}$ that are in different symmetry sectors do not give rise to divergent Berry curvature, despite the resonant denominator in the Kubo formula, due to cancellation of 
matrix elements in the numerator. In our studies, this is circumvented by choosing finite lattices in which the relevant states happen to fall in the same symmetry sector. The generic situation of a physical system with finite disorder that breaks all symmetries naturally falls into this category as well. On the other hand, topological phase transitions can also occur without a gap closing whatsoever [36]. We have not observed this situation in any of the systems we have studied. The above limitations notwithstanding, the BCRG approach provides a useful characterization tool for the detection and classification of topological phase transitions into and out of states of interacting systems characterized by a nontrivial quantum Hall response, as will be shown below in Sec. IV B.

\section{B. Application to FCI-to-trivial transitions}

\section{Peak-divergence scenario}

The triangular and checkerboard lattice models realize the peak-divergence scenario described in Assumption 2.1 of Sec. IV A since the Berry curvature peaks at one or multiple HSPs. Each such peak in the Berry curvature, such as the one shown in the top panel of Fig. 3(b), is well fitted by the $\mathrm{OZ}$ ansatz (4.2). The Berry curvature (2.2b) displays a local extremum at $\boldsymbol{\phi}_{\mathrm{c}}$ as a function of $\boldsymbol{\phi}$, holding $\boldsymbol{M}$ fixed and close to $\boldsymbol{M}_{\mathrm{c}}$. This extremum changes from being a local maximum to a local minimum as $\boldsymbol{M}$ is varied across $\boldsymbol{M}_{\mathrm{c}}$, and the Berry curvature $F_{\star}\left(\boldsymbol{\phi}_{\mathrm{c}}, \boldsymbol{M}\right)$ diverges as $\boldsymbol{M} \rightarrow \boldsymbol{M}_{\mathrm{c}}$.

Numerical results of BCRG applied to the triangular and the checkerboard lattice model are shown in Fig. 7. The Berry curvature (2.2b) at the HSPs of the fBZ is calculated by exact diagonalization on a grid over the parameter space of $\boldsymbol{M}$. For the fermionic triangular lattice model at the density $\rho=\frac{1}{3}$ particles per site, we choose the parameter space $\boldsymbol{M}=$ $\left(t_{3}, V_{1}\right)$ where $t_{3}$ is the third-neighbor hopping and $V_{1}$ is the nearest-neighbor repulsion. For the fermionic checkerboard lattice model at the density $\rho=\frac{1}{6}$, the parameter space is $\boldsymbol{M}=\left(V_{2}, \mu_{2}\right)$ where $V_{2}$ is the second-neighbor repulsion and $\mu_{2}$ is a chemical potential imbalance between sublattices $A$ and $B$. The numerical result shows a BCRG flow that correctly captures the phase boundary between FCI and the topologically trivial state, as indicated by the red dots in Figs. 7(a) and 7(d). The triangular-lattice model shows a rich phase diagram, manifesting both stable and unstable fixed points [blue lines in Fig. 7 (a)], as well as a line (green dots) at which $\left.\nabla_{\boldsymbol{M}} F_{\star}\right|_{\boldsymbol{\phi}_{c}, \boldsymbol{M}}$ vanishes and hence higher-order expansion of Eq. (4.4) is required to correctly describe the BCRG equation. Near the fixed point on the phase boundary, the BCRG flow becomes chaotic, and it becomes difficult to extract the phase boundary.

Drawing an analogy to noninteracting systems [33] and to thermodynamic phase transitions (although we are dealing with given finite lattices), we denote the "critical exponents" associated to the divergences in the many-body Berry curvature $F_{\star}$ and the functions $\xi_{1}$ and $\xi_{2}$ entering the $\mathrm{OZ}$ scaling function (4.2) by

$$
\begin{aligned}
F_{\star}\left(\boldsymbol{\phi}_{\mathrm{c}}, \boldsymbol{M}\right) & \propto\left|\boldsymbol{M}-\boldsymbol{M}_{\mathrm{c}}\right|^{-\alpha}, \\
\xi_{1}(\boldsymbol{M}) & \propto\left|\boldsymbol{M}-\boldsymbol{M}_{\mathrm{c}}\right|^{-v_{1}}, \quad \xi_{2}(\boldsymbol{M}) \propto\left|\boldsymbol{M}-\boldsymbol{M}_{\mathrm{c}}\right|^{-v_{2}} .
\end{aligned}
$$
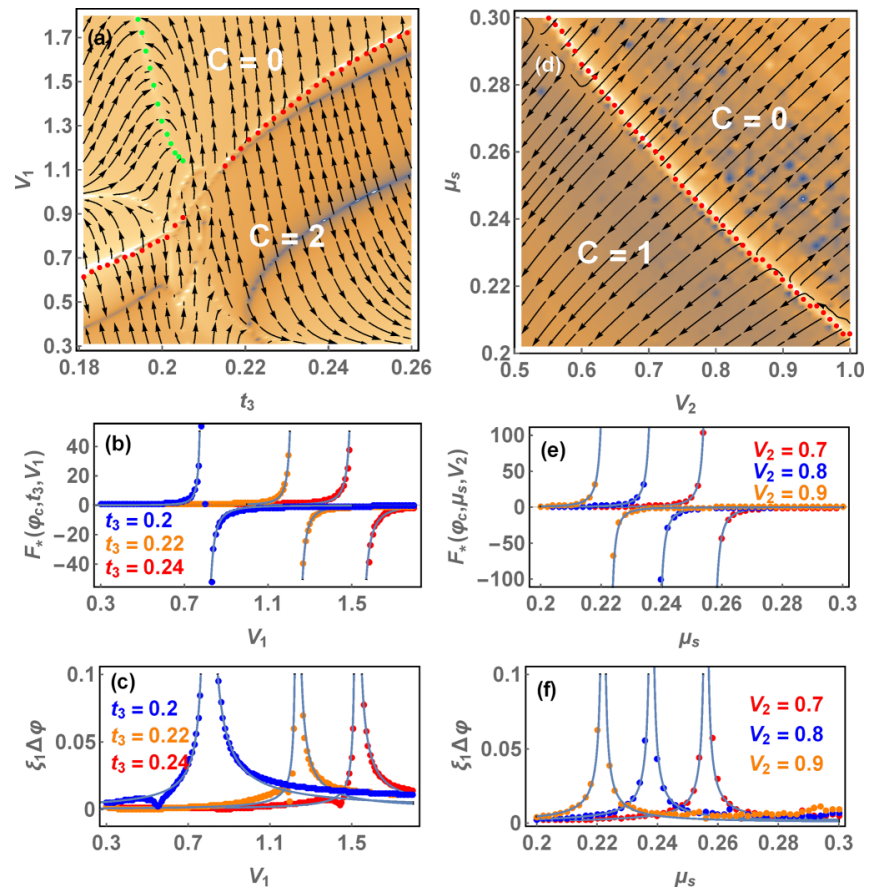

FIG. 7. (a) BCRG flow for the triangular-lattice model using $\Delta \boldsymbol{M}=\left(\Delta t_{3}, \Delta V_{1}\right)=(0.001,0.01)$, and, without loss of generality, choosing $b=0$ in Eq. (4.3), with the topological invariant $C$ labeled for each phase. The flow rate in logarithmic scale is shown by the color code. Blue color indicates a low flow rate and orange a high flow rate. Red dots label the phase boundary, and the green dots the line at which $\left.\nabla_{M} F_{\star}\right|_{\phi_{c}, M}$ in Eq. (4.4) vanishes. The scaling ansatz (4.2) is made to fit the Berry curvature in the neighborhood of the HSP and of a plateau transition. (b), (c) Show the divergence of $F_{\star}\left(\phi_{\mathrm{c}}, t_{3}, V_{1}\right)$ and $\xi_{1}$ versus $V_{1}$ at a few selected $t_{3}$. (d) BCRG flow of the checkerboard model using $\Delta \boldsymbol{M}=\left(\Delta V_{2}, \Delta \mu_{2}\right)=(0.01,0.002)$. (e), (f) Show the divergence of $F_{\star}\left(\boldsymbol{\phi}_{\mathrm{c}}, V_{2}, \mu_{2}\right)$ and $\xi_{1}$ versus $\mu_{2}$ at a few selected $V_{2}$. The small displacement $\Delta \boldsymbol{\phi}=(2 \pi / 1000,0)$ is used for the calculations. Both models feature the same HSP $\boldsymbol{\phi}_{\mathrm{c}}=(0,0)$, and the divergence of $F_{\star}\left(\boldsymbol{\phi}_{\mathrm{c}}, \boldsymbol{M}\right)$ and $\xi_{1}$ are well fitted by the same exponents $\alpha=2$ and $v_{1}=1$, as indicated by the solid lines.

For these two models, the numerical results are well fitted by $\alpha \approx 2$ and $v_{1} \approx v_{2} \approx 1$. This behavior mirrors that of Weyl nodes and satisfies the scaling law $\alpha=v_{1}+v_{2}$ introduced in Appendix B. In Appendix C, we provide a complementary interpretation of $\xi_{i}(\boldsymbol{M})$, with $i=1,2$, in terms of correlation functions on the lattice dual to the fBZ.

\section{Ring-divergence scenario}

The honeycomb model near $V_{2 \mathrm{c}} \approx 1.446$, characterized by quadratic $\boldsymbol{\phi}$ dispersion around $\boldsymbol{\phi}_{c}=(0,0)$, realizes the ringdivergence scenario since the extremum of Berry curvature forms a ring surrounding $\boldsymbol{\phi}_{\mathrm{c}}$. The ring is not necessarily circular, its precise shape is parameter dependent, and the extremum is not uniform along the ring, as can be seen in Fig. 5.

The "critical" behavior of the Berry curvature in this scenario is that, as $\boldsymbol{M} \rightarrow \boldsymbol{M}_{\mathrm{c}}$, (i) the extremum of the Berry curvature diverges, (ii) the radius of the ring along which the Berry curvature reaches its extremal value vanishes, 

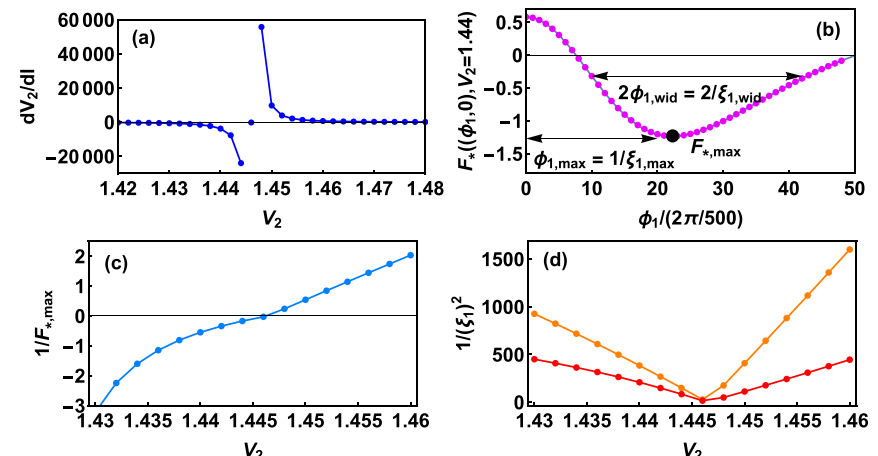

FIG. 8. (a) BCRG flow of the tuning parameter $V_{2}$ for the honeycomb lattice model, obtained using Eq. (4.4) with the choice $b=2$, the grid spacing $\Delta \boldsymbol{\phi}=(3 \pi / 250,0)$, and $\Delta V_{2}=0.002$. Plotted in units of $\Delta V_{2} / \Delta \phi^{2}$, the BCRG flow signals the existence of a quantum critical point at $V_{2 \mathrm{c}} \approx 1.446$ in the thermodynamic limit. (b) Schematics of extracting the two correlation lengths $\xi_{1, \max }$ and $\xi_{1 \text {,wid }}$ from the Berry curvature (purple) along the $\widehat{\boldsymbol{\phi}}_{1}$ direction. (c) The inverse $1 / F_{\star, \max }$ of the Berry curvature extremum $F_{\star \text {, max }}$ versus $V_{2}$. The values at $V_{2}>V_{2 \mathrm{c}}$ are enlarged 200 times for readability. The linear behavior near $V_{2 c}$ corresponds to the exponent $\alpha \approx 1$. (d) Inverses $1 / \xi_{1, \max }^{2}$ and $1 / \xi_{1 \text {,wid }}^{2}$ of the squared correlation lengths $\xi_{1 \text {,max }}^{2}$ (orange) and $\xi_{1 \text {,wid }}^{2}$ (red) versus $V_{2}$ plotted in units of $(\pi / 500)^{2}$. The $1 / \xi_{1 \text {,wid }}^{2}$ at $V_{2}>V_{2 \mathrm{c}}$ is enlarged by 10 times for readability. The linear behavior near $V_{2 \mathrm{c}}$ corresponds to the critical exponent $v \approx \frac{1}{2}$.

(iii) and $F_{\star}\left(\boldsymbol{\phi}_{\mathrm{c}}, \boldsymbol{M}\right)$ remains finite. The extremum changes from a maximum to a minimum as $\boldsymbol{M}$ passes $\boldsymbol{M}_{\mathrm{c}}$. Based on these features, we propose the same scaling procedure [Eq. (4.3)], but with the choice $b>1$ to obtain the BCRG flow, as shown schematically in Fig. 6(b). The scaling procedure gradually reduces the magnitude of the extremum and increases the radius of the ring, and hence the system is gradually flowing away from the critical point, as explained in Appendix A. The BCRG equation follows Eq. (4.4) with $b>1$, while the critical point and fixed point are identified from the direction of the RG flow and Eq. (4.5).

Applying Eq. (4.4) to the honeycomb model, choosing $b>1$, yields the BCRG flow shown in Fig. 8(a) that signals the existence of a quantum critical point in the thermodynamic limit. The ring shape of the Berry curvature implies two scales in the fBZ that represent the radius and the width of the ring. They are extracted in the following manner. Along a particular high-symmetry line, for instance $\widehat{\boldsymbol{\phi}}_{1}$, the extremum of the Berry curvature and its location are denoted by $F_{\star, \max }$ and $\phi_{1, \max }$, respectively. Defining the half-extremum as $\left[F_{\star \text {,max }}+F_{\star}\left(\phi_{\mathrm{c}}, V_{2}\right)\right] / 2$, the half-distance between the two $\phi_{1}$ 's at which the half-maximum occurs is denoted by $\phi_{1 \text {,wid }}$, as shown schematically in Fig. 8(b). In Appendix $C$, we demonstrate that $\xi_{1, \max }:=1 / \phi_{1, \text { max }}$ and $\xi_{1 \text {,wid }}:=1 / \phi_{1 \text {,wid }}$ represent the two length scales over which a correlation function oscillates and decays, respectively. Figures 8(c) and 8(d) provide supporting evidence for the scaling behaviors $F_{\star, \text { max }} \propto\left|V_{2}-V_{2 \mathrm{c}}\right|^{-1}$ and $\xi_{1, \max } \propto \xi_{1 \text {,wid }} \propto$ $\left|V_{2}-V_{2 \mathrm{c}}\right|^{-1 / 2}$. The corresponding scaling exponents are in full agreement with those of noninteracting double Weyl nodes [33].

\section{SUMMARY AND CONCLUSION}

We have investigated precursor signs of quantum phase transitions taking place in the thermodynamic limit between topologically trivial and nontrivial correlated phases by performing detailed numerical studies of interacting twodimensional fermionic and bosonic models harboring FCI states with twisted boundary conditions. The main result of this work is the observation that, when inversion or other pointgroup symmetries are present, the many-body Berry curvature develops divergences at one or more HSPs in the space of twists in the boundary conditions, i.e., the Brillouin zone for twisted boundary conditions (fBZ), for critical values of the parameters in our models defined on finite lattices. Concomitantly, we find nodal points in the dispersion of many-body energy levels in the fBZ at the same HSPs. We observe a connection between the number and dispersion of these nodal points and the many-body Chern number characterizing the states that partake in each transition. The many-body energy levels close to the nodal points can be classified heuristically by the power law that determines the dispersion in $\phi$ space, in analogy to the topological charge of Weyl nodes for the noninteracting Bloch bands of semimetals. We have determined the exponents $p_{d}$ of the dispersion around the nodal points encountered at a number of topological-to-trivial phase transitions in models harboring FCI states. At all these points, $p_{d}$ is an integer. Finally, we have exploited the above observations to develop a scaling approach for the many-body Berry curvature around the HSPs in the $\mathrm{fBZ}$ where it becomes divergent. This procedure is shown to give precursor signs of the thermodynamic phase boundaries of topological phase transitions driven by either interaction or single-particle parameters, and we have empirically found different classes of "criticality," depending again on the exponents $p_{d}$.

Even though the validity of our methods and observations has been verified through a battery of rigorous numerical tests for a number of models, this work is predominantly phenomenological, in the sense that we do not attempt to prove a number of points, in particular (i) whether the ansatz for the scaling of the Berry curvature is unique, and (ii) what the importance of our findings is in the thermodynamic limit. We hope to clarify (at least a subset of) the above points in future work. Finally, our approach can be generalized to the spin quantum Hall effect (see Ref. [14]).

\section{ACKNOWLEDGMENTS}

S.K. is grateful to G. Palumbo for stimulating discussions. S.K. was partially supported through the Boston University Center for Non-Equilibrium Systems and Computation. M.S., W.C., and T.N. acknowledge financial support from a grant of the Swiss National Science Foundation.

\section{APPENDIX A: DEVIATION-REDUCTION MECHANISM}

The scaling hypothesis takes the differential form captured by Eq. (4.4) for the Berry curvature $F_{\star}(\boldsymbol{\phi}, \boldsymbol{M})$. According to this hypothesis, a flow away from the critical point takes place. We are going to derive a different scaling equation upon approaching the fixed-point Berry curvature $F_{\star}^{(\mathrm{f})}\left(\boldsymbol{\phi}, \boldsymbol{M}_{\mathrm{f}}\right)$ that 
is defined by demanding that it carries the Chern number

$$
C_{\star}=\int_{0}^{2 \pi} \int_{0}^{2 \pi} \frac{d \phi_{1} d \phi_{2}}{(2 \pi)^{2}} F_{\star}^{(\mathrm{f})}\left(\boldsymbol{\phi}, \boldsymbol{M}_{\mathrm{f}}\right),
$$

while it obeys the scaling form [compare with Eq. (4.3)]

$$
F_{\star}^{(\mathrm{f})}\left(\boldsymbol{\phi}_{\mathrm{c}}+\delta \boldsymbol{\phi}, \boldsymbol{M}_{\mathrm{f}}\right)=F_{\star}^{(\mathrm{f})}\left(\boldsymbol{\phi}+\delta \boldsymbol{\phi}^{\prime}, \boldsymbol{M}_{\mathrm{f}}\right)
$$

for some $\delta \boldsymbol{\phi}^{\prime}$ about the HSP $\boldsymbol{\phi}_{\mathrm{c}}$ in the fBZ. We make the additive decomposition

$$
F_{\star}(\boldsymbol{\phi}, \boldsymbol{M})=F_{\star}^{(\mathrm{f})}\left(\boldsymbol{\phi}, \boldsymbol{M}_{\mathrm{f}}\right)+\delta F_{\star}^{(\mathrm{f})}(\boldsymbol{\phi}, \boldsymbol{M}),
$$

where

$$
\begin{aligned}
\delta F_{\star}^{(\mathrm{f})}(\boldsymbol{\phi}, \boldsymbol{M})= & \sum_{\boldsymbol{m} \in \mathbb{Z}^{2},|\boldsymbol{m}|>0} \delta F_{\star}^{(\mathrm{f})}(\boldsymbol{m}, \boldsymbol{M}) \\
& \times \cos \left(m_{1} \phi_{1}\right) \cos \left(m_{2} \phi_{2}\right)
\end{aligned}
$$

so that

$$
0=\int_{0}^{2 \pi} \int_{0}^{2 \pi} \frac{d \boldsymbol{\phi}_{1} d \boldsymbol{\phi}_{2}}{(2 \pi)^{2}} \delta F_{\star}^{(\mathrm{f})}(\boldsymbol{\phi}, \boldsymbol{M}),
$$

while we assume the scaling relation

$$
\delta F_{\star}^{(\mathrm{f})}\left(\boldsymbol{\phi}_{\mathrm{c}}+\delta \boldsymbol{\phi}, \boldsymbol{M}\right)=\delta F_{\star}^{(\mathrm{f})}\left(\boldsymbol{\phi}_{\mathrm{c}}+\delta \boldsymbol{\phi}^{\prime}, \boldsymbol{M}^{\prime}\right)
$$

for some pair $\delta \boldsymbol{\phi}^{\prime}$ and $\boldsymbol{M}^{\prime}$. If we choose

$$
\boldsymbol{\phi}_{\mathrm{c}}=\mathbf{0}
$$

and assume the linear relation

$$
\delta \boldsymbol{\phi}^{\prime}=b \delta \boldsymbol{\phi}
$$

for the real number $0 \leqslant b$, we then find the relation

$$
\begin{aligned}
& \delta F_{\star}^{(\mathrm{f})}\left(\boldsymbol{\phi}_{\mathrm{c}}+\delta \boldsymbol{\phi}^{\prime}, \boldsymbol{M}^{\prime}\right)-\delta F_{\star}^{(\mathrm{f})}\left(\boldsymbol{\phi}_{\mathrm{c}}+\delta \boldsymbol{\phi}^{\prime}, \boldsymbol{M}\right) \\
& \quad=\frac{1}{2}\left(\delta \boldsymbol{\phi}-b \delta \boldsymbol{\phi}^{\prime}\right) \cdot\left(\frac{\partial}{\partial \boldsymbol{\phi}} \delta F_{\star}^{(\mathrm{f})}\left(\boldsymbol{\phi}_{\mathrm{c}}+\delta \boldsymbol{\phi}, \boldsymbol{M}\right)\right)
\end{aligned}
$$

to leading order in an expansion in powers of $\delta \phi$. Contrary to Eq. (4.4), this equation is of first order in the derivative with respect to the twisting angles.

The change of $\delta F_{\star}^{(\mathrm{f})}$ as $\boldsymbol{M}$ changes to $\boldsymbol{M}^{\prime}$, holding $\boldsymbol{\phi}_{\mathrm{c}}+\delta \boldsymbol{\phi}^{\prime}$ fixed in the fBZ, is proportional to the derivative of $\delta F_{\star}^{(\mathrm{f})}$ with respect to the twisting angle at $\boldsymbol{\phi}_{\mathrm{c}}+\delta \boldsymbol{\phi}$ in the fBZ holding $\boldsymbol{M}$ fixed. Now, the decomposition (A3) implies that $\delta F_{\star}^{(\mathrm{f})}$ carries the singularity encoded in $F_{\star}$ upon crossing $\boldsymbol{M}_{\mathrm{c}}$. This observation has the following consequences.

For the peak-divergence scenario (in which case $0 \leqslant b<$ $1)$, as one can deduce from Fig. 6(a), when the extremum $F_{\star}\left(\phi_{\mathrm{c}}, \boldsymbol{M}\right)$ is a maximum, then the derivative of $\delta F_{\star}^{(\mathrm{f})}$ is negative whereas the multiplicative prefactor $\left(1-b^{2}\right) / 2>0$ is positive. If so, the left-hand side of Eq. (A6) is negative and the Berry curvature divergence is reduced under the mapping $\boldsymbol{M} \rightarrow \boldsymbol{M}^{\prime}$.

For the ring-divergence scenario (in which case $1<b$ ), when the extremum $F_{\star}\left(\boldsymbol{\phi}_{\mathrm{c}}, \boldsymbol{M}\right)$ is captured by a double Lorentzian with a maximum as shown in Fig. 6(b), then the derivative of $\delta F_{\star}^{(\mathrm{f})}$ is positive whereas the multiplicative prefactor $\left(1-b^{2}\right) / 2<0$ is negative. If so, the left-hand side of Eq. (A6) is negative and the Berry curvature divergence is also reduced under the mapping $\boldsymbol{M} \rightarrow \boldsymbol{M}^{\prime}$. If the extrema of many-body Berry curvature are minima instead of maxima, the same logic leads to a positive left-hand side of Eq. (A6), so the negative divergence of Berry curvature is again reduced.

We conclude that the divergence of the many-body Berry curvature is reduced under this scaling procedure. The system is gradually flowing away from the value $\boldsymbol{M}_{\mathrm{c}}$ at which the Berry curvature diverges.

\section{APPENDIX B: A SCALING LAW IN THE PEAK-DIVERGENCE SCENARIO}

In the peak-divergence scenario of Sec. IV B 1, it is instructive to calculate the contribution

$$
\begin{aligned}
C_{\star \mathrm{div}}(\boldsymbol{M}):= & \int_{-\xi_{1}^{-1}}^{+\xi_{1}^{-1}} \frac{d \delta \phi_{1}}{2 \pi} \int_{-\xi_{2}^{-1}}^{+\xi_{2}^{-1}} \frac{d \delta \phi_{2}}{2 \pi} \\
& \times \frac{F_{\star}\left(\boldsymbol{\phi}_{\mathrm{c}}, \boldsymbol{M}\right)}{1+\xi_{1}^{2}(\boldsymbol{M}) \delta \phi_{1}^{2}+\xi_{2}^{2}(\boldsymbol{M}) \delta \phi_{2}^{2}}
\end{aligned}
$$

to the many-body Chern number that arises from the proximity of $\boldsymbol{\phi}_{\mathrm{c}}+\delta \boldsymbol{\phi}$ to $\boldsymbol{\phi}_{\mathrm{c}}$, as approximated by the OZ ansatz. Here, we assume that $F_{\star}\left(\boldsymbol{\phi}_{\mathrm{c}}, \boldsymbol{M}\right)$ and $\xi_{i}(\boldsymbol{M})$, with $i=1,2$, obey the singular scalings (4.6a) and (4.6b), respectively. It is

$$
C_{\star \operatorname{div}}(\boldsymbol{M})=\text { const } \times \frac{F_{\star}\left(\boldsymbol{\phi}_{\mathrm{c}}, \boldsymbol{M}\right)}{\xi_{1}(\boldsymbol{M}) \xi_{2}(\boldsymbol{M})} .
$$

Since the left-hand side is always finite, although not necessarily constant, on either side of $\boldsymbol{M}_{\mathrm{c}}$, the divergence of $\lim _{\boldsymbol{M} \rightarrow \boldsymbol{M}_{\mathrm{c}}} F_{\star}\left(\boldsymbol{\phi}_{\mathrm{c}}, \boldsymbol{M}\right)$ must be compensated by the divergences of $\lim _{\boldsymbol{M} \rightarrow \boldsymbol{M}_{\mathrm{c}}} \xi_{i}(\boldsymbol{M})$. By matching the divergences of the numerator and denominator on the right-hand side, we deduce the Ornstein-Zernike scaling law

$$
\alpha \approx v_{1}+v_{2}
$$

\section{APPENDIX C: WANNIER STATE CORRELATION FUNCTION}

Our starting point is the twisted boundary conditions that span the $\mathrm{fBZ}$

$$
\left\{\left(\phi_{1}, \phi_{2}\right) \mid 0 \leqslant \phi_{i}<2 \pi, \quad i=1,2\right\} .
$$

We do the rescaling of the fBZ defined by

$$
\varphi_{i}:=\frac{1}{L_{i}\left|\boldsymbol{a}_{i}\right|} \phi_{i}, \quad i=1,2
$$

where $\boldsymbol{a}_{i}$ are the basis vector of the finite lattices made of

$$
L_{1} \times L_{2}
$$

repeated unit cells that were considered in Secs. II and III. The rescaled $\mathrm{fBZ}$ has the area

$$
\prod_{i=1}^{2} \frac{2 \pi}{L_{i}\left|a_{i}\right|}
$$

and $\varphi_{i}$ has the units of inverse length. We interpret the rescaled $\mathrm{fBZ}$ as the Brillouin zone of an auxiliary dual lattice of infinite extent spanned by the lattice vectors

$$
\boldsymbol{R}_{\boldsymbol{m}}:=\sum_{i=1}^{2} m_{i} L_{i} \boldsymbol{a}_{i}, \quad \boldsymbol{m} \equiv\left(m_{1}, m_{2}\right) \in \mathbb{Z}^{2} .
$$


We can associate the Fock space of the many-body Hamiltonians $\widehat{H}$ defined in Sec. III to each site $\boldsymbol{R}_{\boldsymbol{m}}$. Any one of the Hamiltonians $\widehat{H}$ considered in Sec. III has a GS $\left|\Psi_{\star}(\boldsymbol{\phi}, \boldsymbol{M})\right\rangle$ with the wave function in position space

$$
\Psi_{\star}\left(\boldsymbol{r}_{1}, \ldots, \boldsymbol{r}_{N} \mid \boldsymbol{\phi}, \boldsymbol{M}\right):=\left\langle\boldsymbol{r}_{1}, \ldots, \boldsymbol{r}_{N} \mid \Psi_{\star}(\boldsymbol{\phi}, \boldsymbol{M})\right\rangle .
$$

By assumption, this GS carries a nonvanishing many-body Chern number

$$
C_{\star}(\boldsymbol{M}):=\int_{0}^{2 \pi} \int_{0}^{2 \pi} \frac{d \phi_{1} d \phi_{2}}{(2 \pi)^{2}} F_{\star}(\boldsymbol{\phi}, \boldsymbol{M}),
$$

where the Berry curvature $F_{\star}(\boldsymbol{\phi}, \boldsymbol{M})$ was defined in Eq. (2.2c) in some region of parameter space $\left\{\boldsymbol{M} \in \mathbb{R}^{m}\right\}$. For notational simplicity, we shall denote with

$$
\Psi_{\star}\left(\boldsymbol{r}_{1}, \ldots, \boldsymbol{r}_{N} \mid \boldsymbol{\varphi}, \boldsymbol{M}\right):=\left\langle\boldsymbol{r}_{1}, \ldots, \boldsymbol{r}_{N} \mid \Psi_{\star}(\boldsymbol{\varphi}, \boldsymbol{M})\right\rangle
$$

the function obtained by expressing $\phi$ in terms of $\varphi$ in Eq. (C6). With the help of Eq. (2.1), we have the relation

$$
\begin{array}{r}
\Psi_{\star}\left(\boldsymbol{r}_{1}+\boldsymbol{R}_{\boldsymbol{m}}, \ldots, \boldsymbol{r}_{N}+\boldsymbol{R}_{\boldsymbol{m}} \mid \boldsymbol{\varphi}, \boldsymbol{M}\right) \\
=e^{i N \boldsymbol{\varphi} \cdot \boldsymbol{R}_{m}} \Psi_{\star}\left(\boldsymbol{r}_{1}, \ldots, \boldsymbol{r}_{N} \mid \boldsymbol{\varphi}, \boldsymbol{M}\right)
\end{array}
$$

for any site $\boldsymbol{R}_{\boldsymbol{m}}$ of the lattice dual to the rescaled fBZ that was defined in Eq. (C5). If we define

$$
\boldsymbol{X}_{\boldsymbol{m}}:=N \boldsymbol{R}_{\boldsymbol{m}},
$$

we recognize that

$$
\begin{gathered}
\Psi_{\star}\left(\boldsymbol{r}_{1}+N^{-1} \boldsymbol{X}_{\boldsymbol{m}}, \ldots, \boldsymbol{r}_{N}+N^{-1} \boldsymbol{X}_{\boldsymbol{m}} \mid \boldsymbol{\varphi}, \boldsymbol{M}\right) \\
=e^{i \boldsymbol{\varphi} \cdot \boldsymbol{X}_{\boldsymbol{m}}} \Psi_{\star}\left(\boldsymbol{r}_{1}, \ldots, \boldsymbol{r}_{N} \mid \boldsymbol{\varphi}, \boldsymbol{M}\right)
\end{gathered}
$$

takes the form of Bloch's theorem, whereby the center of mass

$$
\boldsymbol{X}:=\sum_{i=1}^{N} \boldsymbol{r}_{i}
$$

of the $N$ quantum particles has been translated by $\boldsymbol{X}_{\boldsymbol{m}}$. Correspondingly, we define the center-of-mass operator

$$
\widehat{\boldsymbol{X}}:=\sum_{i=1}^{N} \widehat{\boldsymbol{r}}_{i}
$$

together with the Wannier state supported on the lattice dual to the rescaled $\mathrm{fBZ}$ that is defined by

$$
\begin{aligned}
\left|W_{\star}\left(\boldsymbol{X}_{\boldsymbol{m}}, \boldsymbol{M}\right)\right\rangle:= & \left(\prod_{i=1}^{2} \int_{0}^{2 \pi /\left(L_{i}\left|\boldsymbol{a}_{i}\right|\right)} \frac{d \varphi_{i}}{2 \pi /\left(L_{i}\left|\boldsymbol{a}_{i}\right|\right)}\right) \\
& \times e^{+i \boldsymbol{\varphi} \cdot\left(\widehat{\boldsymbol{X}}-\boldsymbol{X}_{\boldsymbol{m}}\right)}\left|\Psi_{\star}(\boldsymbol{\varphi}, \boldsymbol{M})\right\rangle .
\end{aligned}
$$

Inversion of Eq. (C14) gives

$$
\left|\Psi_{\star}(\boldsymbol{\varphi}, \boldsymbol{M})\right\rangle=\sum_{\boldsymbol{m} \in \mathbb{Z}^{2}} e^{-i \boldsymbol{\varphi} \cdot\left(\widehat{\boldsymbol{X}}-\boldsymbol{X}_{\boldsymbol{m}}\right)}\left|W_{\star}\left(\boldsymbol{X}_{\boldsymbol{m}}, \boldsymbol{M}\right)\right\rangle .
$$

By construction, the Wannier wave function is defined to be

$$
\begin{aligned}
W_{\star} & \left(\boldsymbol{r}_{1}, \ldots, \boldsymbol{r}_{N} \mid \boldsymbol{X}_{\boldsymbol{m}}, \boldsymbol{M}\right) \\
:= & \left\langle\boldsymbol{r}_{1}, \ldots, \boldsymbol{r}_{N} \mid W_{\star}\left(\boldsymbol{X}_{\boldsymbol{m}}, \boldsymbol{M}\right)\right\rangle \\
= & \left(\prod_{i=1}^{2} \int_{0}^{2 \pi /\left(L_{i}\left|\boldsymbol{a}_{i}\right|\right)} \frac{d \varphi_{i}}{2 \pi /\left(L_{i}\left|\boldsymbol{a}_{i}\right|\right)}\right) \\
& \times e^{+i \boldsymbol{\varphi} \cdot\left(\boldsymbol{X}-\boldsymbol{X}_{\boldsymbol{m}}\right)} \Psi_{\star}\left(\boldsymbol{r}_{1}, \ldots, \boldsymbol{r}_{N} \mid \boldsymbol{\varphi}, \boldsymbol{M}\right) .
\end{aligned}
$$

The expectation value of the center-of-mass operator $\widehat{\boldsymbol{X}}$ in the Wannier state $\left|W_{\star}\left(\boldsymbol{X}_{\boldsymbol{m}}, \boldsymbol{M}\right)\right\rangle$ is as localized as may be about $\boldsymbol{X}_{\boldsymbol{m}}$ in the lattice dual to the rescaled fBZ. The Fourier transform of the many-body Berry curvature defined by Eq. (2.2c) is denoted

$$
\begin{aligned}
F_{\star \boldsymbol{X}_{\boldsymbol{m}}}(\boldsymbol{M}):= & \left(\prod_{i=1}^{2} \int_{0}^{2 \pi /\left(L_{i}\left|\boldsymbol{a}_{i}\right|\right)} \frac{d \varphi_{i}}{2 \pi /\left(L_{i}\left|\boldsymbol{a}_{i}\right|\right)}\right) \\
& \times e^{+i \boldsymbol{\varphi} \cdot \boldsymbol{X}_{m}} F_{\star}(\boldsymbol{\varphi}, \boldsymbol{M}) .
\end{aligned}
$$

It is represented in terms of the Wannier state by the correlation function [37-39]

$$
F_{\star \boldsymbol{X}_{m}}(\boldsymbol{M})=-\mathrm{i}\left\langle W_{\star}\left(\boldsymbol{X}_{\boldsymbol{m}}, \boldsymbol{M}\right)\left|\left(X_{m 1} \widehat{X}_{2}-X_{m 2} \widehat{X}_{1}\right)\right| W_{\star}(\mathbf{0}, \boldsymbol{M})\right\rangle .
$$

If we use the $\mathrm{OZ}$ ansatz

$$
F_{\star}\left(\boldsymbol{\varphi}_{\mathrm{c}}+\delta \boldsymbol{\varphi}, \boldsymbol{M}\right) \sim \frac{F_{\star}\left(\boldsymbol{\varphi}_{\mathrm{c}}, \boldsymbol{M}\right)}{1+\sum_{i=1}^{2}\left[\xi_{i}(\boldsymbol{M}) L_{i}\left|\boldsymbol{a}_{i}\right|\right]^{2} \delta \varphi_{i}^{2}}
$$

to fit the peak-divergence scenario, we then identify the characteristic width $\left[\xi_{i}(\boldsymbol{M}) L_{i}\left|\boldsymbol{a}_{i}\right|\right]^{-1}$ of $F_{\star}(\boldsymbol{\varphi}, \boldsymbol{M})$ as a function of $\varphi$ in the rescaled fBZ. Upon Fourier transform of the OZ ansatz (C19), the correlation function $F_{\star \boldsymbol{X}_{m}}(\boldsymbol{M})$ has the characteristic decay length in the lattice dual to the rescaled fBZ given by $\xi_{i}(\boldsymbol{M}) L_{i}\left|\boldsymbol{a}_{i}\right|$. In the ring-divergence scenario, the Fourier transform of the ring-shaped Berry curvature in Fig. 8(b) gives a correlation function $F_{\star X_{m}}(\boldsymbol{M})$ that oscillates with $\xi_{\max }(\boldsymbol{M}) L_{i}\left|\boldsymbol{a}_{i}\right|$ and decays with $\xi_{\text {wid }}(\boldsymbol{M}) L_{i}\left|\boldsymbol{a}_{i}\right|$.

This Wannier state formalism provides an appealing interpretation for the singularities of the Berry curvature in the fBZ for finite systems. As detailed in Sec. II, the choice of twisted boundary conditions in the continuous range $[0,2 \pi) \times[0,2 \pi)$ forms an auxiliary space that allows for the definition of the Chern number. In the thermodynamic limit, this Chern number becomes the quantized Hall conductivity. At the same time, imposing these twisted boundary conditions on many-body wave functions as in Eq. (C11) allows for a definition of an infinite lattice dual to the fBZ. On this infinite lattice, which should not be confused with the original finite lattice $\Lambda$ on which the many-body Hamiltonians have been diagonalized, the functions $\xi_{i}(\boldsymbol{M}) L_{i}\left|\boldsymbol{a}_{i}\right|$ with $i=1,2$, take the meaning of correlation lengths. Moreover, the Fourier transform over the rescaled fBZ of the Berry curvature $F_{\star}(\boldsymbol{\varphi}, \boldsymbol{M})$ that delivers $F_{\star X_{m}}(\boldsymbol{M})$ can be interpreted as a a correlation function that measures the overlap of Wannier states centered at two copies of the finite lattice $\Lambda$ that are distance $\boldsymbol{X}_{\boldsymbol{m}}$ away on this dual lattice. It is in this respect that the many-body Berry curvature for any exact eigenstate of the many-body Hamiltonian defined on the finite lattice $\Lambda$ is similar to scaling functions in critical phenomena. The same is true when identifying the divergence of the $\xi(\boldsymbol{M})$ 's at $\boldsymbol{M}_{\mathrm{c}}$ and their vanishing at $\boldsymbol{M}_{\mathrm{f}}$ with the notion of scale invariance.

For noninteracting tight-binding Hamiltonians for which Bloch's theorem holds upon imposing periodic boundary conditions on a finite lattice, using twisted boundary conditions allows to explore the Brillouin zone in the thermodynamic limit. It is for this reason that the critical behavior of the Berry curvature for the finite number of occupied states in the valence 
band gives access to some thermodynamic critical exponents [33-35].

For interacting Hamiltonians defined on a finite lattice $\Lambda$, the critical behavior of the Berry curvature needs to be related to the quantum phase transition in the thermodynamic limit. To prove or disprove such a relation, it is necessary to perform finite-size scaling numerically, which is not possible with any numerical method available today. A diverging correlation length corresponds to a critical finite-size Wannier many-body state. A large correlation length is interpreted as the finite-size interacting system being close to a gap-closing many-body level crossing. A short correlation length implies the finite-size interacting system is deep inside a gapped (trivial or nontrivial) topological phase. This is irrespective of whether the quantum phase transition involves a change in a Landau order parameter or not.

\section{APPENDIX D: SYMMETRIES OF MANY-BODY HAMILTONIANS IN FLUX SPACE}

In this appendix we show how spatial symmetries of an interacting tight-binding Hamiltonian $\widehat{H}(\mathbf{0})$, present when the (finitely sized) system is defined on a lattice with periodic boundary conditions, translate into symmetries of $\widehat{H}(\phi)$ in flux space when twisted boundary conditions $\phi$ are imposed.

Consider a symmetry operation $\widehat{S}$ and a tight-binding Hamiltonian with a density-density interaction

$$
\begin{aligned}
\widehat{H}(\boldsymbol{\phi})= & \sum_{\boldsymbol{k} \in \mathrm{BZ}} \sum_{\alpha, \beta} \widehat{c}_{\boldsymbol{k}, \alpha}^{\dagger} \mathcal{H}_{\boldsymbol{k}+\boldsymbol{\phi}, \alpha, \beta} \widehat{c}_{\boldsymbol{k}, \beta} \\
& +\sum_{\boldsymbol{i}, \boldsymbol{j} ; \alpha, \beta} V_{\boldsymbol{i}, \boldsymbol{j} ; \alpha, \beta} \widehat{n}_{\boldsymbol{i}, \alpha} \widehat{n}_{\boldsymbol{j}, \beta},
\end{aligned}
$$

where $\alpha$ and $\beta$ label local degrees of freedom in the unit cell such as orbitals and spin. Due to its density-density form, the interaction term is unaffected by the boundary conditions, which only affect the quadratic part $\mathcal{H}_{\boldsymbol{k}+\boldsymbol{\phi}, \alpha, \beta}$.

The action of $\widehat{S}$ on the second quantized operators can be represented by a unitary $B_{k ; \alpha, \beta}$ :

$$
\begin{aligned}
\widehat{S} \widehat{c}_{\boldsymbol{k}, \alpha}^{\dagger} \widehat{S}^{-1} & =\sum_{\beta} B_{\boldsymbol{k} ; \alpha, \beta} \widehat{c}_{S(\boldsymbol{k}), \beta}^{\dagger}, \\
\left(\widehat{S} \widehat{c}_{\boldsymbol{k}, \alpha}^{\dagger} \widehat{S}^{-1}\right)^{\dagger} & =\sum_{\beta} B_{\boldsymbol{k} ; \alpha, \beta}^{*} \widehat{c}_{S(\boldsymbol{k}), \beta},
\end{aligned}
$$

where $S(\boldsymbol{k})$ applies the spatial transformation (like a reflection or rotation) to the momentum. By assumption, the system with periodic boundary conditions is invariant under $\widehat{S}$, i.e.,

$$
\widehat{S} \widehat{H}(\mathbf{0}) \widehat{S}^{-1}=\widehat{H}(\mathbf{0}) .
$$

If we assume that $\widehat{S}^{-1}=\widehat{S}^{\dagger}$, this implies

$$
\mathcal{H}_{S(\boldsymbol{k}), \alpha, \beta}=\sum_{\alpha^{\prime}, \beta^{\prime}} B_{\boldsymbol{k} ; \alpha^{\prime}, \alpha} \mathcal{H}_{\boldsymbol{k}, \alpha^{\prime}, \beta^{\prime}} B_{\boldsymbol{k} ; \beta^{\prime}, \beta}^{*}
$$

Using this relation, one shows that

$$
\widehat{S} \widehat{H}(\boldsymbol{\phi}) \widehat{S}^{-1}=\widehat{H}[S(\boldsymbol{\phi})] .
$$

Spatial symmetries thus imply that the Hamiltonians with boundary conditions $\phi$ and $S(\phi)$ are unitarily related. It follows that gauge-invariant quantities like the many-body Berry curvature obey the same symmetries, even in finite-size systems

$$
F_{n}(\boldsymbol{\phi}, \boldsymbol{M})=F_{n}(S(\boldsymbol{\phi}), \boldsymbol{M}) .
$$

[1] J. von Neumann and E. P. Wigner, Über das verhalten von eigenwerten bei adiabatischen prozessen, in The Collected Works of Eugene Paul Wigner: Part A: The Scientific Papers, edited by A. S. Wightman (Springer, Berlin, 1993), pp. 294-297.

[2] C. Herring, Phys. Rev. 52, 365 (1937).

[3] E. Blount, Solid State Phys. 13, 305 (1962).

[4] M. V. Berry and M. Wilkinson, Proc. R. Soc. London, Ser. A 392, 15 (1984).

[5] G. E. Volovik, The Universe in a Helium Droplet (Clarendon, Oxford, 2003).

[6] Q. Niu, D. J. Thouless, and Y. S. Wu, Phys. Rev. B 31, 3372 (1985).

[7] T. Fukui, Y. Hatsugai, and H. Suzuki, J. Phys. Soc. Jpn. 74, 1674 (2005).

[8] T. Neupert, L. Santos, C. Chamon, and C. Mudry, Phys. Rev. Lett. 106, 236804 (2011).

[9] N. Regnault and B. A. Bernevig, Phys. Rev. X 1, 021014 (2011).

[10] D. J. Thouless, M. Kohmoto, M. P. Nightingale, and M. den Nijs, Phys. Rev. Lett. 49, 405 (1982).

[11] D. J. Thouless, Phys. Rev. B 40, 12034 (1989).

[12] R. B. Laughlin, Phys. Rev. B 23, 5632 (1981).

[13] C. N. Varney, K. Sun, M. Rigol, and V. Galitski, Phys. Rev. B 84, 241105 (2011).
[14] T.-S. Zeng, W. Zhu, J.-X. Zhu, and D. N. Sheng, arXiv:1708.03538.

[15] A. C. M. Carollo and J. K. Pachos, Phys. Rev. Lett. 95, 157203 (2005).

[16] S.-L. Zhu, Phys. Rev. Lett. 96, 077206 (2006).

[17] D. N. Sheng, X. Wan, E. H. Rezayi, K. Yang, R. N. Bhatt, and F. D. M. Haldane, Phys. Rev. Lett. 90, 256802 (2003).

[18] S. Kourtis, J. W. F. Venderbos, and M. Daghofer, Phys. Rev. B 86, 235118 (2012).

[19] J. W. F. Venderbos, S. Kourtis, J. van den Brink, and M. Daghofer, Phys. Rev. Lett. 108, 126405 (2012).

[20] S. Kourtis, T. Neupert, C. Chamon, and C. Mudry, Phys. Rev. Lett. 112, 126806 (2014).

[21] S. Kourtis and M. Daghofer, Phys. Rev. Lett. 113, 216404 (2014).

[22] K. Sun, Z. Gu, H. Katsura, and S. Das Sarma, Phys. Rev. Lett. 106, 236803 (2011).

[23] D. N. Sheng, Z. Z.-C. Gu, K. Sun, and L. Sheng, Nat. Commun. 2, 389 (2011).

[24] Y.-L. Wu, B. A. Bernevig, and N. Regnault, Phys. Rev. B 85, 075116 (2012).

[25] A. M. Läuchli, Z. Liu, E. J. Bergholtz, and R. Moessner, Phys. Rev. Lett. 111, 126802 (2013). 
[26] F. D. M. Haldane, Phys. Rev. Lett. 61, 2015 (1988).

[27] Y.-F. Wang, Z.-C. Gu, C.-D. Gong, and D. N. Sheng, Phys. Rev. Lett. 107, 146803 (2011).

[28] C. Fang, M. J. Gilbert, X. Dai, and B. A. Bernevig, Phys. Rev. Lett. 108, 266802 (2012).

[29] B. Bradlyn, L. Elcoro, J. Cano, M. G. Vergniory, Z. Wang, C. Felser, M. I. Aroyo, and B. A. Bernevig, Nature (London) 547, 298 (2017).

[30] M. Oshikawa, Phys. Rev. Lett. 84, 1535 (2000).

[31] J. Zhang, C.-M. Jian, F. Ye, and H. Zhai, Phys. Rev. Lett. 105, 155302 (2010).

[32] X. Wan, A. M. Turner, A. Vishwanath, and S. Y. Savrasov, Phys. Rev. B 83, 205101 (2011).
[33] W. Chen, M. Legner, A. Rüegg, and M. Sigrist, Phys. Rev. B 95, 075116 (2017).

[34] W. Chen, J. Phys.: Condens. Matter 28, 055601 (2016).

[35] W. Chen, M. Sigrist, and A. P. Schnyder, J. Phys.: Condens. Matter 28, 365501 (2016).

[36] A. Amaricci, J. C. Budich, M. Capone, B. Trauzettel, and G. Sangiovanni, Phys. Rev. Lett. 114, 185701 (2015).

[37] N. Marzari, A. A. Mostofi, J. R. Yates, I. Souza, and D. Vanderbilt, Rev. Mod. Phys. 84, 1419 (2012).

[38] M. Gradhand, D. V. Fedorov, F. Pientka, P. Zahn, I. Mertig, and B. L. Gyrffy, J. Phys.: Condens. Matter 24, 213202 (2012).

[39] X. Wang, J. R. Yates, I. Souza, and D. Vanderbilt, Phys. Rev. B 74, 195118 (2006). 